\title{
TRPV4 channel opening mediates pressure-induced pancreatitis initiated by Piezo1 activation
}

\author{
Sandip M. Swain, ${ }^{1}$ Joelle M.-J. Romac, ${ }^{1}$ Rafiq A. Shahid, ${ }^{1}$ Stephen J. Pandol, ${ }^{2}$ Wolfgang Liedtke, ${ }^{3}$ Steven R. Vigna, ${ }^{1,4}$ \\ and Rodger A. Liddle1,5 \\ 'Department of Medicine, Duke University, Durham, North Carolina, USA. ${ }^{2}$ Cedars-Sinai Medical Center, Los Angeles, California, USA. ${ }^{3}$ Department of Neurology and ${ }^{4}$ Department of Cell Biology, \\ Duke University, Durham, North Carolina, USA. Department of Veterans Affairs Health Care System, Durham, North Carolina, USA.
}

\begin{abstract}
Elevated pressure in the pancreatic gland is the central cause of pancreatitis following abdominal trauma, surgery, endoscopic retrograde cholangiopancreatography, and gallstones. In the pancreas, excessive intracellular calcium causes mitochondrial dysfunction, premature zymogen activation, and necrosis, ultimately leading to pancreatitis. Although stimulation of the mechanically activated, calcium-permeable ion channel Piezo1 in the pancreatic acinar cell is the initial step in pressure-induced pancreatitis, activation of Piezo1 produces only transient elevation in intracellular calcium that is insufficient to cause pancreatitis. Therefore, how pressure produces a prolonged calcium elevation necessary to induce pancreatitis is unknown. We demonstrate that Piezo1 activation in pancreatic acinar cells caused a prolonged elevation in intracellular calcium levels, mitochondrial depolarization, intracellular trypsin activation, and cell death. Notably, these effects were dependent on the degree and duration of force applied to the cell. Low or transient force was insufficient to activate these pathological changes, whereas higher and prolonged application of force triggered sustained elevation in intracellular calcium, leading to enzyme activation and cell death. All of these pathological events were rescued in acinar cells treated with a Piezo1 antagonist and in acinar cells from mice with genetic deletion of Piezo1. We discovered that Piezo1 stimulation triggered transient receptor potential vanilloid subfamily 4 (TRPV4) channel opening, which was responsible for the sustained elevation in intracellular calcium that caused intracellular organelle dysfunction. Moreover, TRPV4 gene-KO mice were protected from Piezo1 agonist- and pressure-induced pancreatitis. These studies unveil a calcium signaling pathway in which a Piezo1-induced TRPV4 channel opening causes pancreatitis.
\end{abstract}

\section{Introduction}

The pancreas is highly sensitive to pressure (1-3), and elevated pancreatic duct pressure is a major cause of acute pancreatitis. A gallstone impacted at the junction of the pancreatic and common bile ducts increases pancreatic duct pressure and can initiate pancreatitis (4-6). Intraductal pancreatic pressure is also increased during the clinical procedure endoscopic retrograde cholangiopancreatography (ERCP) when radiocontrast dye is injected into the pancreatic duct to visualize the pancreas (7). Acute pancreatitis develops in up to $20 \%$ of high-risk patients undergoing this procedure (8). Mechanical injury to the pancreas through abdominal trauma is also a common cause of pancreatitis (9). Recently, our group demonstrated that the pancreatic acinar cell senses pressure through the mechanically activated ion channel Piezo1 and that activation of Piezo1 on acinar cells by pressure or a Piezo1-specific agonist induced acute pancreatitis (1). Moreover, mice with acinar cell-specific deletion of Piezo1 were protected

\section{Related Commentary: p. 2199}

Conflict of interest: The authors have declared that no conflict of interest exists. Copyright: (5) 2020, American Society for Clinical Investigation.

Submitted: October 7, 2019; Accepted: January 23, 2020; Published: April 13, 2020

Reference information: J Clin Invest. 2020;130(5):2527-2541.

https://doi.org/10.1172/JCI134111. from pressure-induced pancreatitis. Thus, mechanical activation of Piezo1 is sufficient to cause pancreatitis.

Mechanical forces including static pressure, fluid shear stress, and membrane stretch activate Piezo1 channel opening, which allows cations, most notably $\mathrm{Ca}^{2+}$, to flow into the cell (10-14). Recently, Yoda1, a pharmacological agonist selective for Piezo1, but not Piezo2, has been identified (15). Piezo1 is expressed in numerous tissues that respond to shear force, including the vascular endothelium, lung, skin, and urinary bladder $(16,17)$. Studying the signaling of Piezo1 in the pancreas offers an attractive model for unveiling the pathological response because of the uniquely sensitive behavior of pancreatic acinar cells to alterations in intracellular calcium levels.

Intracellular $\mathrm{Ca}^{2+}$ concentrations are tightly regulated in pancreatic acinar cells and provide the major signal for digestive enzyme secretion $(18,19)$. Cholecystokinin (CCK), acetylcholine, and bombesin are pancreatic secretagogues that raise cytosolic calcium $\left(\left[\mathrm{Ca}^{2+}\right]_{i}\right)$ and thus stimulate secretion (20-22). Binding of these agonists to their respective $G$ protein-coupled receptors triggers the release of $\mathrm{Ca}^{2+}$ from the ER through increased activity of phosphoinositide-specific phospholipase $\mathrm{C}$ that cleaves phosphatidylinositol, 4,5-bisphosphate $\left(\mathrm{PIP}_{2}\right)$ to inositol 1,4,5-trisphosphate $\left(\mathrm{IP}_{3}\right)$ and diacylglycerol (23). Notably, this $\mathrm{Ca}^{2+}$ rise is necessary for normal cell signaling. Perturbation of $\mathrm{Ca}^{2+}$ signaling is associated with pancreatic acinar cell death (23). Supramaximal concentrations of agonists such as CCK lead to activation of calcium release-activated channels (CRAC) 
A

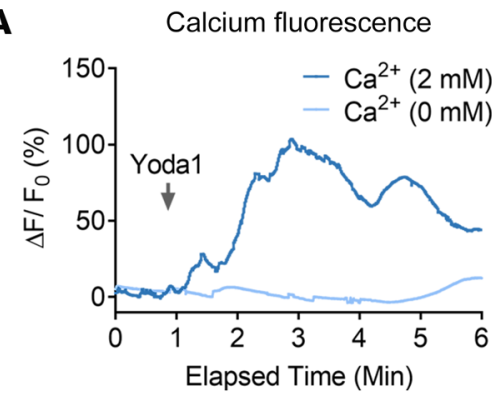

C

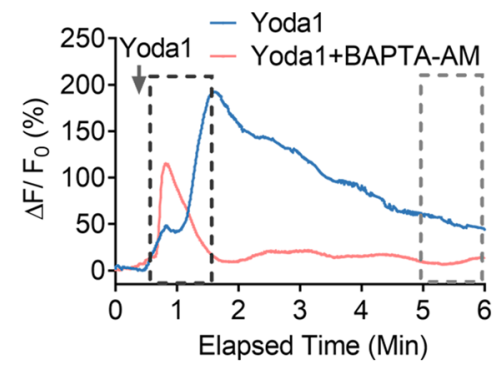

$\mathbf{E}$

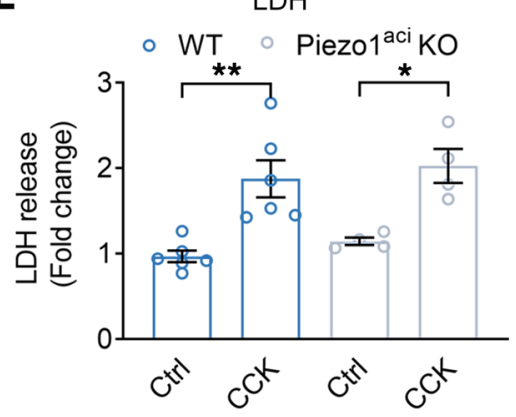

B

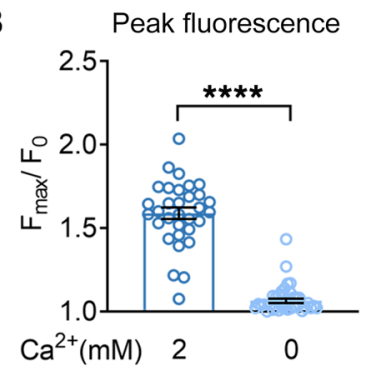

D

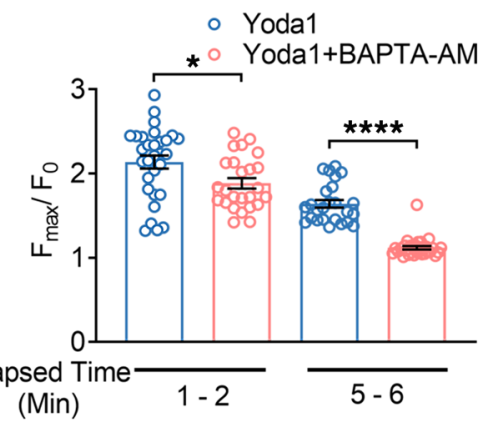

$\mathbf{F}$

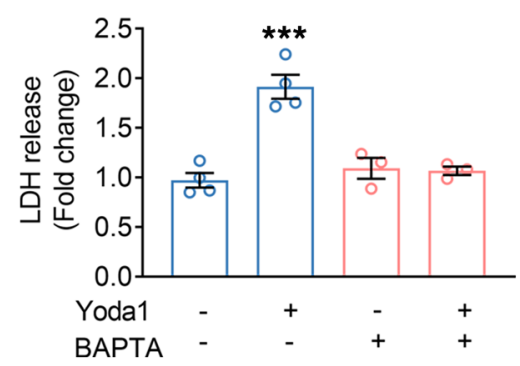

G
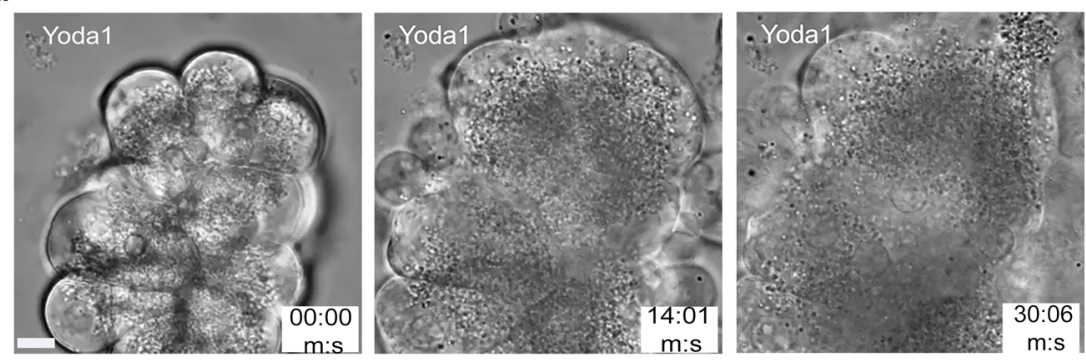

Figure 1. Piezo1 activation increases $\left[\mathrm{Ca}^{2+}\right]_{\mathrm{i}}$ and induces cell death in pancreatic acini. (A) Live-cell imaging of pancreatic acini loaded with calcium 6-QF. Pancreatic acini were incubated with Yoda1 $(25 \mu \mathrm{M})$ in the presence $(2 \mathrm{mM})$ or absence $(0 \mathrm{mM})$ of extracellular calcium. Yoda1 was added at the time indicated by the arrow. (B) Comparison of the Yoda1-induced peak $\left[\mathrm{Ca}^{2+}\right]_{i}$ is expressed as the ratio of peak intensity $\left(F_{\max }\right) /$ baseline intensity $\left(F_{0}\right)$ from 32 to 41 cells. (C) The effects of Yoda1 $(50 \mu \mathrm{M})$ on $\left[\mathrm{Ca}^{2+}\right]_{\mathrm{i}}$ in the absence or presence of the calcium chelator BAPTA-AM $(20 \mu \mathrm{M})$. (D) Statistical analysis of peak $\left(F_{\max } / F_{0}\right)$ from 25 to 30 cells in which acinar cells were preincubated 30 minutes with BAPTA-AM $(20 \mu \mathrm{M})$ before Yoda1 application. $\left(F_{\text {max }} / F_{0}\right)$ was calculated from the time periods 1 to 2 minutes and 5 to 6 minutes to assess the initial transient and sustained $\left[\mathrm{Ca}^{2+}\right]_{i}$ levels, respectively. (E) The effects of CCK $(1 \mathrm{nM})$ on LDH release from isolated pancreatic acini from WT and Piezo $1^{\text {aci }}-K O$ mice from 4 to 6 experiments. (F) The effects of Yoda1 $(50 \mu \mathrm{M})$ on LDH release from acinar cells with and without preincubation of BAPTA-AM for 30 minutes are shown from 3 to 5 experiments. (G) Brightfield images of pancreatic acini at different time points in the presence of Yoda1 $(50 \mu \mathrm{M})$. Images represent a plane (5 $\mu \mathrm{m}$ thick) from a Z-stack of captured images to visualize the changes in cell morphology and granule movement. Images were captured with a $\times 100$ oil objective. Statistical analyses were performed using Student's $t$ test (B, D) and 1-way ANOVA with Tukey's multiple comparison test ( $\mathbf{E}$ and $\mathbf{F}$ ). ${ }^{*} P \leq 0.05$; ${ }^{* *} P \leq 0.01$; ${ }^{* * *} P \leq 0.001,{ }^{* * *} P \leq 0.0001$. Data are shown as mean \pm SEM. Scale bar: $10 \mu \mathrm{m}$. in the plasma membrane (24), resulting in a sustained elevation in $\left[\mathrm{Ca}^{2+}\right]_{\mathrm{i}}$ and intracellular activation of proenzymes in pancreatic acinar cells that ultimately leads to cell death (24). Due to their intensive protein synthesis, acinar cells have high energy requirements and mitochondrial demands. Mitochondrial dysfunction can lead to impairment in energy-requiring processes, including proenzyme sorting, intracellular lysosome and zymogen vesicle formation and stability, and cellular integrity (25). Disruption of any of these functions can lead to pancreatitis. Calcium overload causes opening of the mitochondrial permeability transition pore (MPTP), collapses the mitochondrial membrane potential, and disrupts the proton gradient necessary for ATP production that is required to satisfy the high energy demands of the acinar cell (25-29). Therefore, maintenance of proper mitochondrial function is necessary to prevent pancreatitis.
The calcium-permeable ion channel transient receptor potential vanilloid subfamily 4 (TRPV4) is expressed in various tissues and cells (e.g., smooth muscle of the urinary bladder, epithelial cells of kidney, airways, sensory neurons, and vascular endothelium) and is involved in several physiological processes and diseases, including regulation of blood flow, shear-induced vasodilation, and epithelial ciliary activity (30-37). TRPV4-mediated pathological events include neurogenic inflammation, hyperalgesia, endothelial dysfunction, and pulmonary and cardiac tissue fibrosis $(33,36,38-40)$.

TRPV4 may be activated by physical force (e.g., shear stress, membrane stretching) and hypotonic cell swelling, but this sensing appears to be indirect $(41,42)$. However, the mechanism by which these physical forces activate TRPV4 is not clear. 
Hypotonic cell swelling and shear stress stimulate phospholipase A2 (PLA2) activity (42). In general, elevated PLA2 activity triggers the release of arachidonic acid (AA) and its metabolite, $5^{\prime}, 6^{\prime}$-epoxyeicosatrienoic acid (5',6'-EET) (43). 5',6'-EET is an endogenous ligand that can activate TRPV4 (43). However, the mechanism by which physical stimuli activate PLA2 is not known. Phosphorylation of PKA and PKC increases the sensitization of TRPV4 $(44,45)$; therefore, it is possible that activation of TRPV4 through AA may require PKA or PKC phosphorylation (44).

Here, we demonstrate that activation of Piezo1 in pancreatic acinar cells by direct chemical stimulation and fluid shear stress produced a sustained increase in cytosolic $\mathrm{Ca}^{2+}$, followed by mitochondrial depolarization and premature trypsinogen activation. Moreover, Piezo1-induced $\mathrm{Ca}^{2+}$ signaling is distinctly different from CCK-induced $\mathrm{Ca}^{2+}$ signaling, suggesting that multiple pathways may contribute to the initiation of pancreatitis. To explain how Piezo1 activation led to sustained and pathological elevation in $\left[\mathrm{Ca}^{2+}\right]_{\mathrm{i}}$, we explored other potential calcium-entry pathways and discovered that both mouse and human pancreatic acinar cells express the calcium-permeable channel TRPV4. We also demonstrate that Piezo1 induced the activation of PLA2, which causes TRPV4 channel opening.

\section{Results}

Piezo1 induces sustained cytoplasmic $\mathrm{Ca}^{+}$elevation and cell death. In order to determine whether calcium signaling is responsible for the effects of Piezo1 on the pancreas, we first examined the ability of the chemical Piezo1 agonist Yoda1 to regulate intracellular calcium $\left(\left[\mathrm{Ca}^{2+}\right]_{\mathrm{i}}\right)$ in freshly isolated pancreatic acini. Changes in $\left[\mathrm{Ca}^{2+}\right]_{\mathrm{i}}$ were determined in acinar cells loaded with the calcium indicator calcium 6-QF. We recently reported that the Piezo1 antagonist GsMTx4 inhibits the Yoda1-mediated $\left[\mathrm{Ca}^{2+}\right]_{\mathrm{i}}$ rise in pancreatic acini $(1,46)$. Here, we demonstrate the contribution of external and intracellular free calcium on Piezo1-mediated $\left[\mathrm{Ca}^{2+}\right]_{i}$. Yoda1 in the presence of bath calcium $(2 \mathrm{mM})$ produced an initial transient $\mathrm{Ca}^{2+}$ rise, followed by sustained intracellular calcium elevation (Figure 1, A and B, and Supplemental Video 1; supplemental material available online with this article; https://doi.org/10.1172/ JCI134111DS1). Removal of external calcium completely abolished the Yoda1-mediated $\left[\mathrm{Ca}^{2+}\right]_{\mathrm{i}}$ rise (Figure 1, A and B). However, preincubating cells with BAPTA-AM (a cell-permeable $\mathrm{Ca}^{2+}$ chelator) for 30 minutes abolished the sustained $\mathrm{Ca}^{2+}$ rise (Figure 1, C and D). $\mathrm{CCK}$ is a secretagogue that stimulates pancreatic enzyme secretion by increasing $\left[\mathrm{Ca}^{2+}\right]_{\mathrm{i}}$. In contrast to the effects on Yoda1-induced $\left[\mathrm{Ca}^{2+}\right]_{\mathrm{i}}$, preincubation of cells with BAPTA-AM completely eliminated the effect of CCK on $\left[\mathrm{Ca}^{2+}\right]_{\mathrm{i}}$ (Supplemental Figure 1).

To examine the effect of sustained Piezo1 activation on $\left[\mathrm{Ca}^{2+}\right]_{i}$ and its relation to cellular injury, we treated pancreatic acini with Yoda1 and cellular injury was assessed by measuring lactate dehydrogenase $(\mathrm{LDH})$ release. Cells were preincubated with or without BAPTA-AM. Chelating intracellular free calcium with BAPTA-AM protected pancreatic acini from Yoda1-induced LDH release (Figure 1F). We confirmed the specificity of these effects for Piezo1 by comparing the cytotoxic effects of CCK (Figure 1E). At high concentrations, CCK is well known to cause cell damage in vitro and pancreatitis in vivo $(47,48)$. As shown in Figure 1E, CCK produced comparable cell damage in pancreatic acini from both WT mice and mice with selective genetic deletion of Piezo1 in pancreatic acinar cells (Piezo aci-KO mice) (1). We visualized the effect of Yoda1 on pancreatic acini over time by live-cell imaging. Application of Yoda1 $(50 \mu \mathrm{M})$ caused swelling of WT pancreatic acinar cells and release of zymogen granules from the basolateral surface and gradually ruptured the cell membrane (Figure $1 \mathrm{G}$ and Supplemental Video 2). Pancreatic acinar cells from Piezo1 ${ }^{\text {aci-KO }}$ mice did not respond to Yoda1 (Supplemental Video 3).

In pancreatic acinar cells, CCK at supraphysiological concentrations produces a sustained elevation of $\left[\mathrm{Ca}^{2+}\right]_{\mathrm{i}}$, the initial phase of which is due to release of $\mathrm{Ca}^{2+}$ from the ER (23). Following this initial rise, the sustained phase occurs through the activation of CRAC, which allows extracellular $\mathrm{Ca}^{2+}$ to flow into cells. In order to determine whether the Piezo1-mediated sustained $\left[\mathrm{Ca}^{2+}\right]_{i}$ elevation is due to CRAC activation, we examined the effects of the CRAC inhibitor CM4620, which selectively inhibits Orai, the main component of CRAC (49). Preincubating acinar cells with CM4620 for 1 hour blocked the sustained elevation in $\left[\mathrm{Ca}^{2+}\right]_{i}$ produced by CCK (100 and 1000 pM) (Supplemental Figure 2, A-C). Notably, CM4620 did not completely block the $\left[\mathrm{Ca}^{2+}\right]_{\mathrm{i}}$ rise induced by either concentration of CCK, and a residual calcium wave was always observed following CCK despite CM4620 administration (Supplemental Figure 2, A-C). This persistent calcium wave was possibly due to $\mathrm{Ca}^{2+}$ released from ER stores $(50,51)$. In contrast to the effects on CCK-stimulated $\left[\mathrm{Ca}^{2+}\right]_{\mathrm{i}}, \mathrm{CM} 4620$ did not alter the rise in $\left[\mathrm{Ca}^{2+}\right]_{\mathrm{i}}$ following Yoda1 stimulation (Supplemental Figure 2, $\mathrm{D}$ and $\mathrm{E}$ ), indicating that CRAC channels are not the source of sustained $\left[\mathrm{Ca}^{2+}\right]_{\mathrm{i}}$ elevation following Piezo1 activation.

To determine whether Piezo1 gene deletion altered the acinar cell response to secretagogue stimulation, we examined the effects of CCK on $\left[\mathrm{Ca}^{2+}\right]_{i}$ in pancreatic acini from Piezo1 ${ }^{\text {aci }}-\mathrm{KO}$

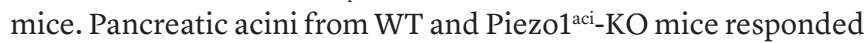
equally to both physiological (20 pM) and supraphysiological (1 nM) CCK concentrations (Supplemental Figure 3, A-D). In order to confirm that Piezo1 and CCK stimulate $\left[\mathrm{Ca}^{2+}\right]_{\mathrm{i}}$ through distinct mechanisms, we first activated Piezo1 channels in pancreatic acini through mechanical force by applying a blunt glass pipette to the surface of acinar cells to a depth of $5 \mu \mathrm{m}$ for 1 second. This blunt pushing produced a transient $\left[\mathrm{Ca}^{2+}\right]_{i}$ elevation. Cells were then exposed to CCK (20 pM). Slight mechanical pushing did not alter the sensitivity of pancreatic acini to subsequent CCK exposure (Supplemental Figure 3, E and F). In light of the finding that Piezo1 and CCK affect $\left[\mathrm{Ca}^{2+}\right]_{\mathrm{i}}$ through separate pathways, we sought to determine whether together Yoda1 and CCK accentuated the deleterious effects on pancreatic acinar cells. Compared with individual application of Yoda1 and CCK, acinar cell death was greater when cells were exposed to both Yoda1 and CCK (Supplemental Figure 3, G and H).

Piezo1 agonist Yoda1 induces pathological events in pancreatic acini. Pancreatic acinar cells possess abundant mitochondria and are highly metabolically active. Proper mitochondrial function is critical for cellular homeostasis, and mitochondrial dysfunction has been implicated in the pathogenesis of pancreatitis. We postulated that the elevations in cytoplasmic $\left[\mathrm{Ca}^{2+}\right]$ that were observed with Yoda1 stimulation and Piezo1 activation in pancreatic acinar cells may affect mitochondrial depolarization. To test this hypothesis, we used a cell-permeable and mitochondrial-potential sensitive fluorescent dye, tetramethyl rhodamine ester (TMRE) $(52,53)$. 
A

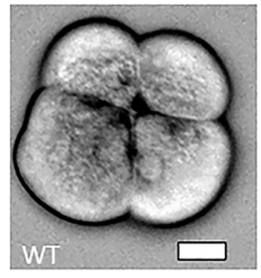

Mitochondrial depolarization (TMRE fluorescence)
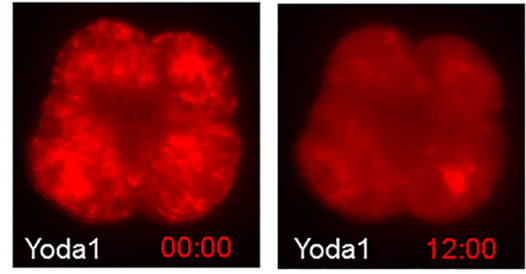

Decrease in TMRE fluorescence

C

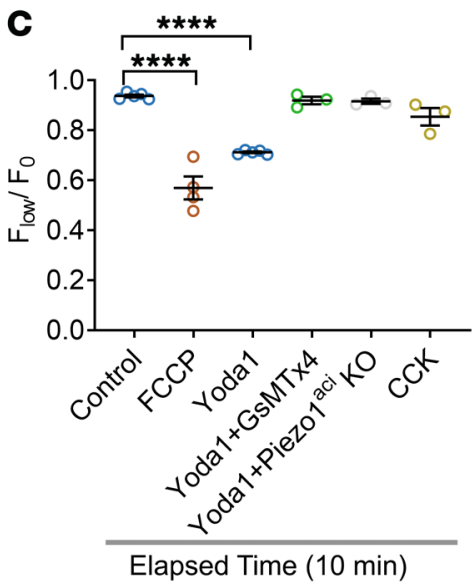

$\mathbf{F}$
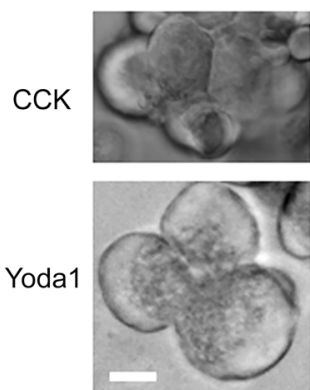

G

BZiPAR fluorescence

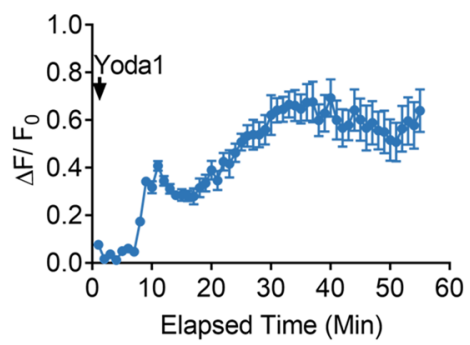

D

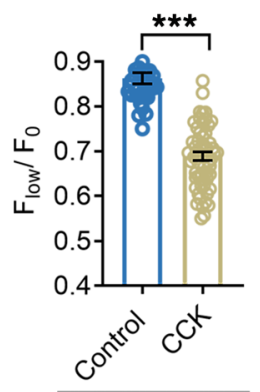

Elapsed Time (20 min)
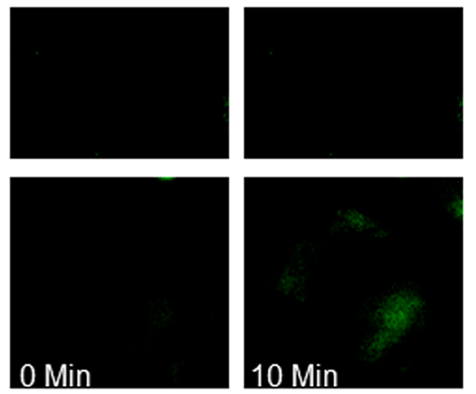

H Peak fluorescence ○ WT

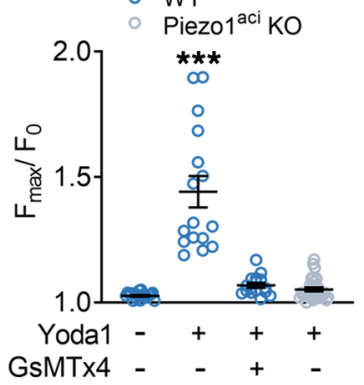

B

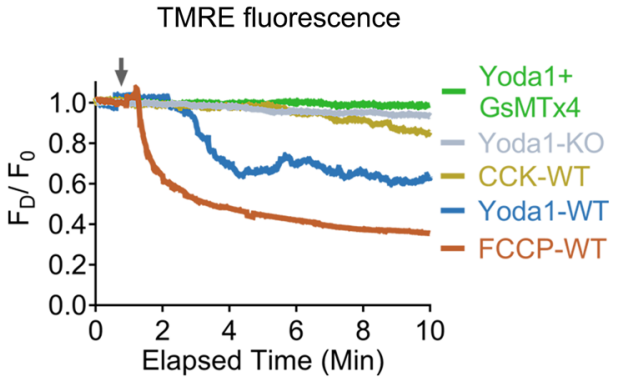

$\mathbf{E}$

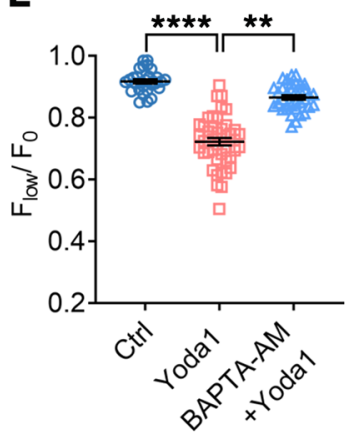

Trypsinogen activation (BZiPAR fluorescence)
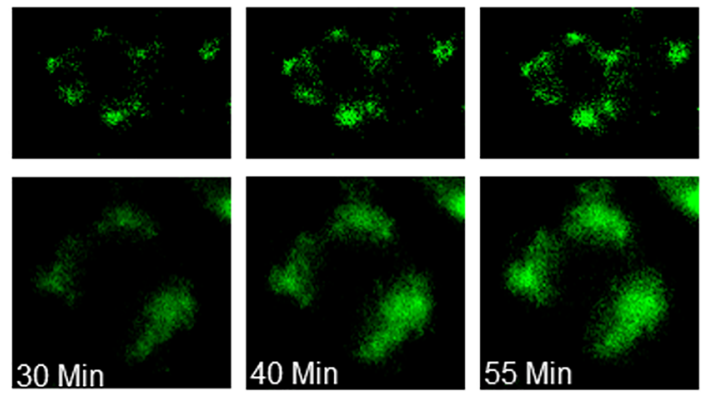

I Peak fluorescence

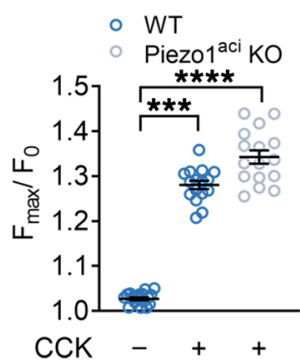

Figure 2. Piezo1 activation induces mitochondrial depolarization and trypsinogen activation in pancreatic acini. (A) Brightfield and fluorescence images of TMRE-loaded pancreatic acini 0 and 12 minutes after Yoda1 $(50 \mu \mathrm{M})$ application. (B) Traces of live-cell TMRE fluorescence intensity of single acinar cells over time following the administration of Yoda1 (arrow). $F_{D}$ is the decrease in TMRE fluorescence, and $F_{0}$ is the baseline TMRE intensity. Acini from WT or

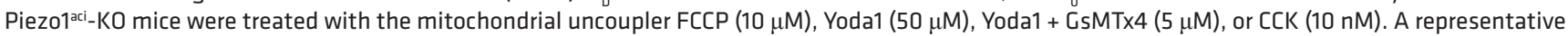
tracing from 3 experiments is shown. (C) Mean decrease in TMRE intensity from $\mathbf{B}$ is depicted. $n=3-5$ independent experiments with $15-20$ cells in each experiment. (D) Decrease in TMRE intensity of pancreatic acini is shown in response to CCK (10 nM) from 60 cells over 20 minutes. (E) Decrease in fluorescence intensity of TMRE in pancreatic acinar cells upon Yoda1 application with or without preincubation of BAPTA-AM (20 $\mu$ M) for 20 minutes is shown from $29-45$ cells. (F) Live-cell imaging of intracellular trypsin activation with CCK (10 nM) and Yoda1 $(50 \mu M)$ treatments. Scale bar: $10 \mu \mathrm{m}$. (C) Time course of Yoda1-induced trypsin activation is shown for 3 experiments. (H) Peak BZiPAR fluorescence was measured in acini from WT mice treated with Yoda1 in the absence or presence of GsMTx4 (5 $\mu \mathrm{M})$ or in acini from Piezo1aci-KO mice. $n=3-5$ independent experiments with $16-31$ cells. (I) Pancreatic acini from

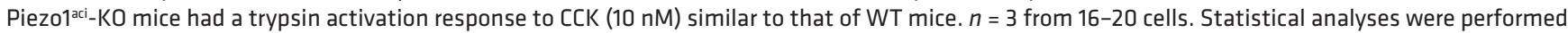
using Student's $t$ test (D) and 1-way ANOVA (C, E, H, and I). Data are shown as mean \pm SEM. ${ }^{* *} P \leq 0.01 ;{ }^{* *} P \leq 0.001 ;{ }^{* * *} P \leq 0.0001$. 
A
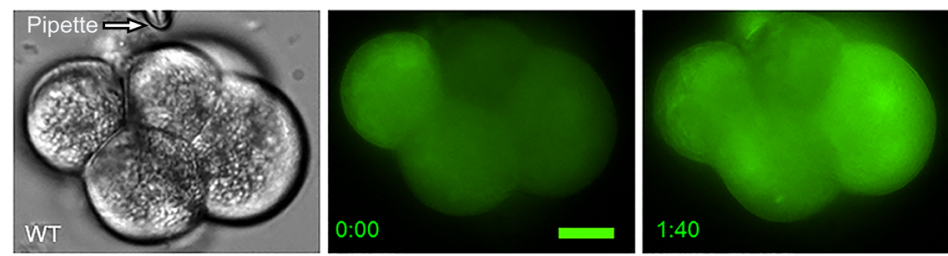

B

Calcium fluorescence
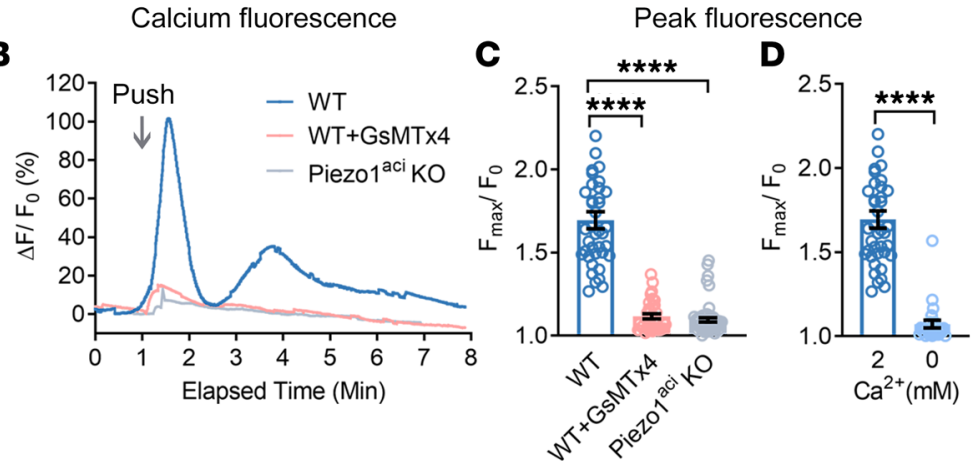

$\mathbf{E}$
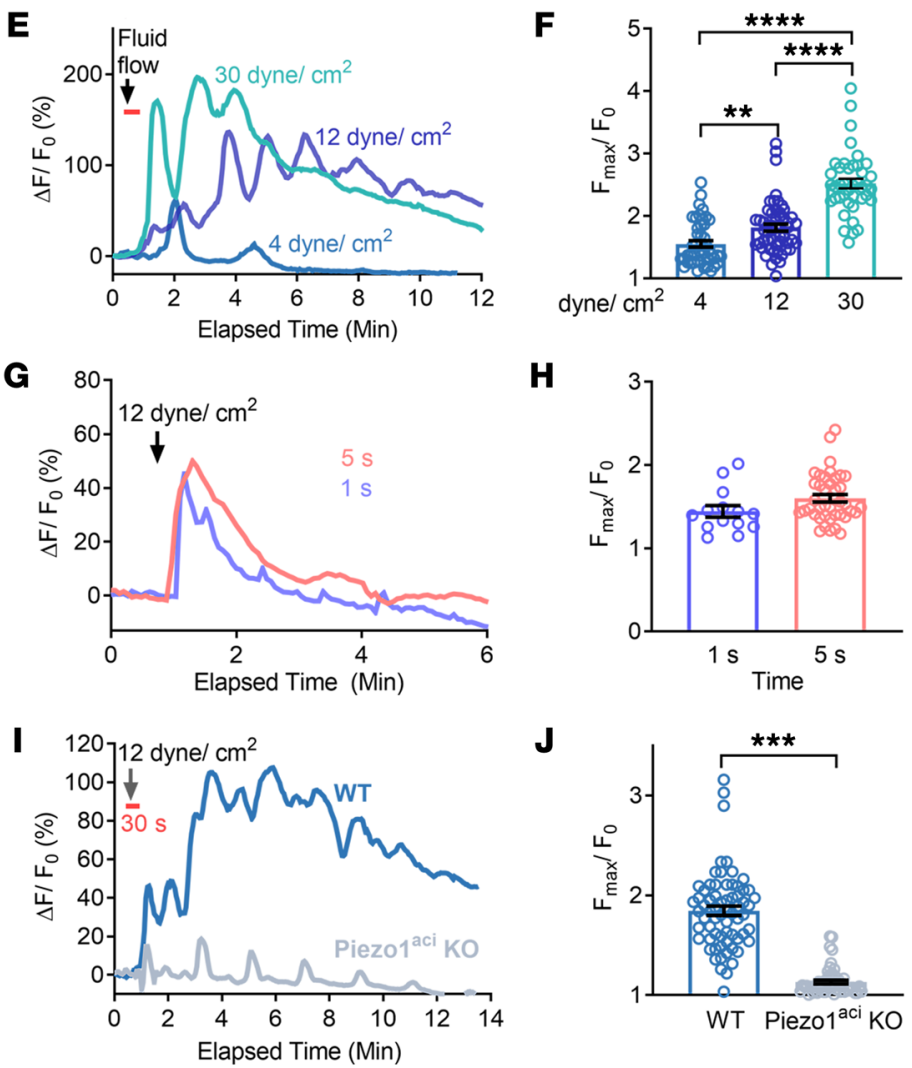

\section{H}

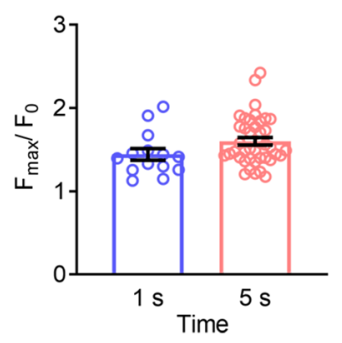

$\mathbf{J}$

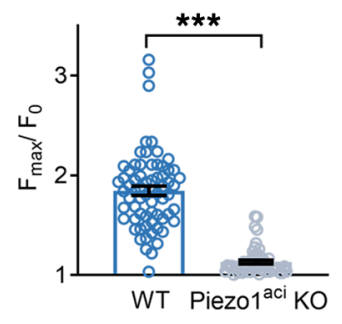

Figure 3. Mechanical pushing and fluid shear stress increase $\left[\mathrm{Ca}^{2+}\right]_{i}$ in pancreatic acini. (A) Brightfield and live-cell imaging of pancreatic acini loaded with calcium 6-QF at time 0 and at time of maximum fluorescence 1:40 (min:s) after mechanical pushing. Scale bars: $10 \mu \mathrm{m}$. (B) Representative $\left[\mathrm{Ca}^{2+}\right]_{i}$ fluorescence tracings from single acinar cells in an acinus during the course of mechanical pushing with a blunt pipette. (C) Peak $\left[\mathrm{Ca}^{2+}\right]_{i}$ levels following mechanical pushing of acini from WT or Piezo1aci-KO mice. (D) Blunt pushing with a micropipette increased $\left[\mathrm{Ca}^{2+}\right]$ fluorescence only in the presence of extracellular $\mathrm{Ca}^{2+}$. Results represent data from 25-39 cells in 3 independent experiments. (E) Representative traces for relative fluorescence intensity $\left(\Delta F / F_{0}\right)$ of calcium 6-QF-loaded cells are shown in response to applied shear stress at the forces shown in the graph. The duration of fluid flow shear stress was 30 seconds, as indicated by the orange bar. $(\mathbf{F})$ The average peak $\left[\mathrm{Ca}^{2+}\right]_{i}$ intensity of $\mathrm{F}_{\max } /$ $\mathrm{F}_{0}$ is shown for $41-54$ cells. $n=3-4$ independent experiments. (C and $\mathbf{H})$ The relative fluorescence intensity $\left(\Delta F / F_{0}\right)$ and average peak $\left[\mathrm{Ca}^{2+}\right]_{i}$ intensity of pancreatic acini in response to 12 dyne/ $\mathrm{cm}^{2}$ shear stress for 1 or 5 seconds. Data in $\mathbf{H}$ are averaged from 14-41 cells. (I) Representative traces of relative fluorescence intensity $\left(\Delta F / F_{0}\right)$ from calcium 6-QF-loaded WT and Piezo1-KO acinar cells before and after applying $12 \mathrm{dyne} / \mathrm{cm}^{2}$ shear stress for 30 seconds. (J) Average peak intensity of $F_{\max } / F_{0}$ from the experiment depicted in I. Data represent a total of 53-67 cells and 3 independent experiments. Statistical analyses were performed using Student's $t$ test (D, H, and $\mathbf{J})$ and 1-way ANOVA with Tukey's multiple comparison (C and $\mathbf{F}$ ). Data are shown as mean \pm SEM. ${ }^{* *} P \leq 0.01 ;{ }^{* *} P \leq 0.001 ;{ }^{* * *} P \leq 0.0001$.

intracellular-free calcium (Figure 2E). We observed that Yoda1-induced mitochondrial depolarization occurred at a higher rate than that induced with CCK $(10 \mathrm{nM})$ (Figure 2, A-D). Yoda1-mediated mitochondrial depolarization occurred within 3 minutes of application; however, CCK-mediated mitochondrial depolarization occurred more slowly and was observed only after 10 minutes of drug exposure.

Premature zymogen activation and autodigestion of acinar cells are critical events in pancreatitis. Moreover, pathophysiological elevations in cytoplasmic $\left[\mathrm{Ca}^{2+}\right]$ have been associated with intracellular trypsinogen activation $(52,54)$. We postulated that prolonged activation of Piezo1 may cause trypsinogen activation through a calcium-mediated pathway. We visualized realtime trypsinogen activation in pancreatic acini using a trypsin-sensitive, cell-permeable fluorescent probe, rhodamine 110, bis(CBZ-L-isoleucyl-L-prolyl-L-arginine amide) (BZiPAR), which becomes fluorescent once

TMRE accumulates as punctate distributions in mitochondria due to high negative membrane potential. The fluorescence intensity of this punctate pattern declines following mitochondrial depolarization. Application of Yoda1 $(50 \mu \mathrm{M})$ throughout the period of livecell imaging significantly decreased TMRE fluorescence intensity indicative of depolarization of the mitochondria (Figure 2, A-C). GsMTx4 blocked the Yoda1-induced mitochondrial depolarization in WT cells. Yoda1 did not depolarize mitochondria in Piezo1 ${ }^{\text {aci }}-\mathrm{KO}$ cells (Figure 2, B and C). Pancreatic acinar cells preincubated with BAPTA-AM for 20 minutes were protected from Yoda1-induced mitochondrial depolarization, suggesting a significant role for it is cleaved specifically by trypsin $(52,55)$. Application of either Yoda1 $(50 \mu \mathrm{M})$ or CCK $(10 \mathrm{nM})$ activated trypsin in pancreatic acini over time (Figure 2, F and G, and Supplemental Video 4). Yoda1 induced trypsin activation within 10 minutes of application; however, CCK stimulated trypsin activation more slowly and was observed only after 20 minutes of drug exposure (Supplemental Video 4). GSMTx4 blocked the Yoda1-induced trypsin activation in pancreatic acinar cells (Figure 2H). Pancreatic acinar cells from

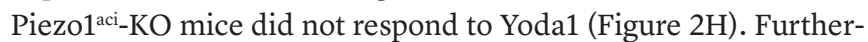
more, CCK-mediated trypsin activation was similar in acini from

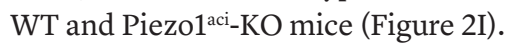


A

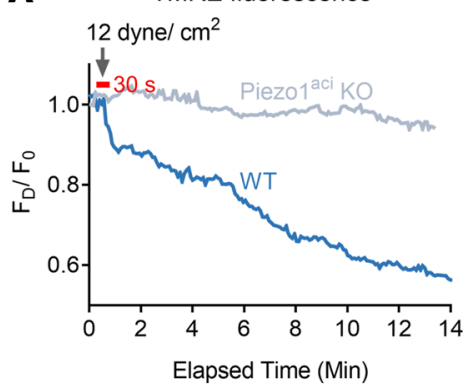

C

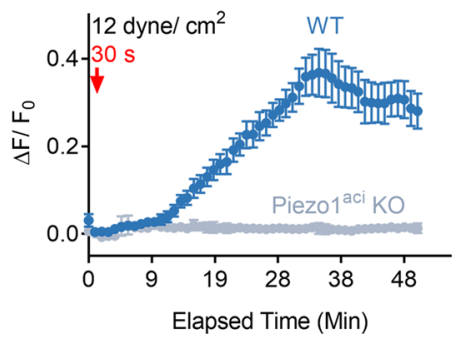

B Decrease in TMRE fluorescence

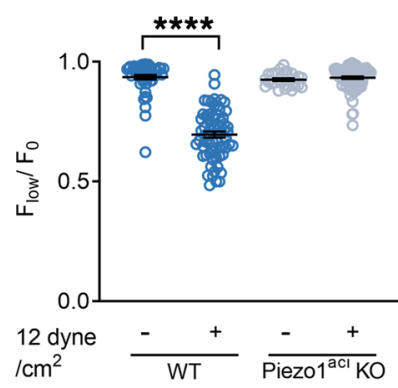

D Peak BZiPAR fluorescence - WT

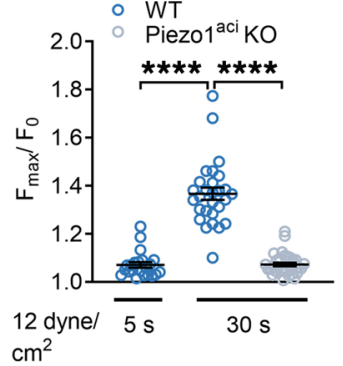

Figure 4. Fluid shear stress induces mitochondrial depolarization and trypsinogen activation in pancreatic acini. (A) Pancreatic acini were subjected to fluid shear force of $12 \mathrm{dyne} / \mathrm{cm}^{2}$, and TMRE dye fluorescence intensity of pancreatic acinar cells from WT and Pie-

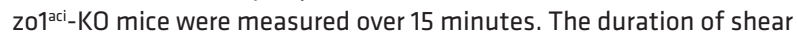
stress for 30 seconds is marked by the orange bar. $F_{0}$ represents the decrease in TMRE fluorescence over time, and $F_{0}$ represents the base line TMRE intensity before fluid shear stress. (B) The mean average decrease in fluorescence intensity of TMRE over 15 minutes is shown. $F_{\text {low }}$ is the lowest TMRE fluorescent intensity after fluid shear stress during imaging. The total number of acinar cells examined were as follows: control (without fluid shear stress) $=56$; fluid shear stress on WT acini = 50; and fluid shear stress on Piezolaci-KO acini $=91$ and WT acini $=67 . n=3$ independent experiments. (C) Traces represent live-cell trypsin activity upon fluid shear stress of 12 dyne $/ \mathrm{cm}^{2}$ for

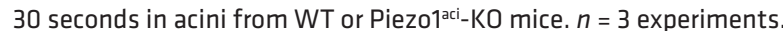
(D) Peak fluorescence intensity of BZiPAR over 50 minutes from 3 experiments; total number of acinar cells were as follows: WT $=28$ and Piezo1ai-KO $=36$. Data are shown as the mean $\pm \mathrm{SEM}$. ${ }^{* * *} P \leq$ 0.0001 . Statistical analyses were performed using Student's $t$ test (B) and 1-way ANOVA with Tukey's multiple comparison (D).
Mechanical pushing- and shear stress-induced $\left[\mathrm{Ca2}^{+}\right]_{i}$ elevation in pancreatic acini. Mechanical activation of Piezo1 in acinar cells was demonstrated by applying a blunt glass pipette to the surface of acinar cells to a depth of $5 \mu \mathrm{m}$ for 1 second. This stimulation produced a transient $\left[\mathrm{Ca}^{2+}\right]_{\mathrm{i}}$ rise (Figure $3, \mathrm{~A}$ and B, and Supplemental Video 5) that was completely blocked by prior treatment of the cells with GsMTx4 (Figure 3C). In addition, mechanical pushing did not increase $\left[\mathrm{Ca}^{2+}\right]_{\mathrm{i}}$ in acinar cells from Piezo1 ${ }^{\text {aci-KO }}$ mice (Figure $3 \mathrm{C}$ and Supplemental Video 6). Removal of external calcium blocked the mechanical pushing-mediated $\left[\mathrm{Ca}^{2+}\right]_{\mathrm{i}}$ rise (Figure 3D). In order to apply physical force for a longer time, we used a fluid shear stress approach to avoid cell damage that might occur with repeated mechanical pushing and technical difficulties. The fluid shear stress approach is similar to the situation in which high fluid pressure is injected into the pancreatic duct, as we have demonstrated (1), which has been used as a model for the clinical condition of excess filling of the pancreatic duct in humans during ERCP (7).

Mechanical shear stress is a physiological activator of Piezo1 in many tissues, including vascular endothelium $(12,13)$. To determine whether Piezo1 channels are responding to fluid shear stress in the pancreas, we evaluated changes in $\left[\mathrm{Ca}^{2+}\right]_{\mathrm{i}}$ in freshly isolated pancreatic acini plated in a shear flow chamber (13). Following a period as brief as 30 seconds, the peak intensity of $\left[\mathrm{Ca}^{2+}\right]_{\mathrm{i}}$ in pancreatic acini increased as greater shear stress forces were applied (Figure 3, E and $\mathrm{F}$ ). Shear stresses of 4,12 , and $30 \mathrm{dyne} / \mathrm{cm}^{2}$ increased the peak $\left[\mathrm{Ca}^{2+}\right]_{\mathrm{i}}$ by $1.5 \pm 0.1-, 1.8 \pm 0.1-$, and $2.5 \pm 0.1$-fold, respectively (Figure $3, \mathrm{E}$ and $\mathrm{F}$ ). The 12 and 30 dyne $/ \mathrm{cm}^{2}$ stresses caused a sustained elevation in $\left[\mathrm{Ca}^{2+}\right]_{\mathrm{i}}$ and mimicked the $\left[\mathrm{Ca}^{2+}\right]_{\mathrm{i}}$ pattern induced by Yoda1 $(25 \mu \mathrm{M}$ and $50 \mu \mathrm{M})$ (Figure 3E and Figure 1, A and C). However, the lower shear stress force of 4 dyne $/ \mathrm{cm}^{2}$ elicited only a transient $\left[\mathrm{Ca}^{2+}\right]_{\mathrm{i}}$ rise (Figure $3 \mathrm{E}$ ) without a prolonged $\left[\mathrm{Ca}^{2+}\right]_{\mathrm{i}}$ elevation. Furthermore, fluid shear stress force of $12 \mathrm{dyne} / \mathrm{cm}^{2}$ applied for $1 \mathrm{sec}-$ ond or 5 seconds was not sufficient to induce a sustained elevation in $\left[\mathrm{Ca}^{2+}\right]_{\mathrm{i}}$ (Figure $3, \mathrm{G}$ and $\mathrm{H}$ ). The sustained $\left[\mathrm{Ca}^{2+}\right]_{\mathrm{i}}$ elevation at 12 dyne $/ \mathrm{cm}^{2}$ did not occur in pancreatic acini from Piezo1 ${ }^{\text {aci-KO mice, }}$ although small transient $\left[\mathrm{Ca}^{2+}\right]_{i}$ peaks at regular intervals could be seen in some cells (Figure 3, I and J). We suspect that small transient spikes in $\left[\mathrm{Ca}^{2+}\right]_{\mathrm{i}}$ occurring at regular intervals could be from low expression of other mechanically sensitive channels.

Fluid shear stress induces pathological events in pancreatic acini. To determine whether fluid shear stress-activated Piezo1 facilitates mitochondrial depolarization, we monitored live-cell mitochondrial depolarization in pancreatic acini loaded with the mitochondrial sequestrant dye TMRE (200 nM) from WT and Pie-

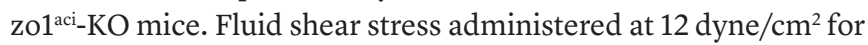
30 seconds that caused a sustained elevation in $\left[\mathrm{Ca}^{2+}\right]_{\mathrm{i}}$ decreased the TMRE intensity over time in WT pancreatic acini, but not in

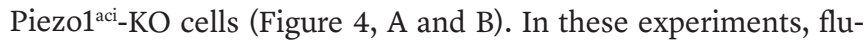
id shear stress led to a sustained depolarization consistent with a state of mitochondrial dysfunction (Supplemental Video 7) (25, 56). The mitochondrial potential of pancreatic acini with Piezo1 deletion was not affected by these conditions of fluid shear stress (Figure 4B and Supplemental Video 7). These results demonstrate that Piezo1 channels mediate fluid shear stress-induced mitochondrial depolarization. In contrast, mechanical pushing of acinar cells with a glass pipette only slightly depolarized the mitochondria in $20 \%$ of acinar cells, while the remainder were completely unaffected. Overall, it appears that pushing of pancreatic acinar cells once for 1 second was not sufficient to substantially depolarize the mitochondria (Supplemental Figure 4A).

Pancreatic enzyme activation in pancreatic acinar cells is a key pathological feature in pancreatitis. As reported here, the Piezo1 agonist Yoda1 induces trypsinogen activation, which is the initial step in activation of other zymogens in the pancreas. To determine whether fluid shear stress mimics the Yoda1 effect and trypsin activation, pancreatic acinar cells were loaded with the trypsin activity-measuring probe BZiPAR. Pancreatic acini were then sub- 
A
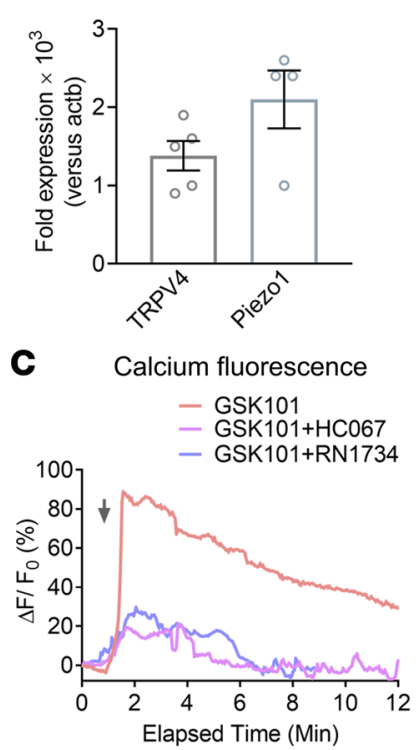

E

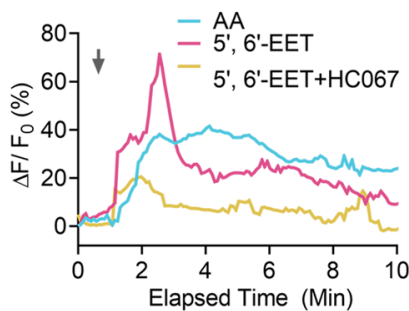

B Immunostaining TRPV4 Nunc blue

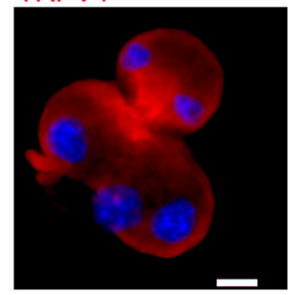

D Peak fluorescence

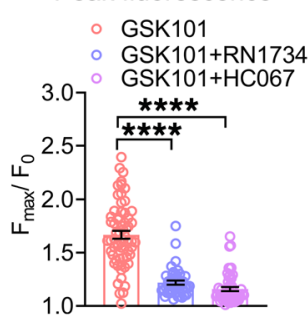

F

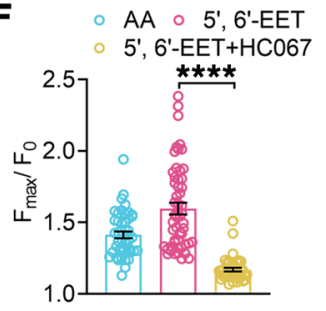

G

Calcium fluorescence

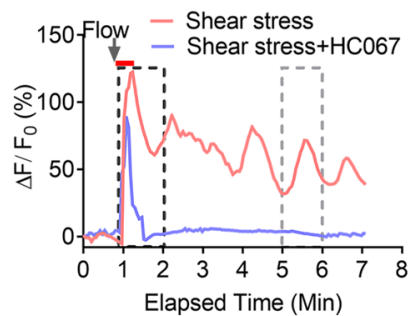

I

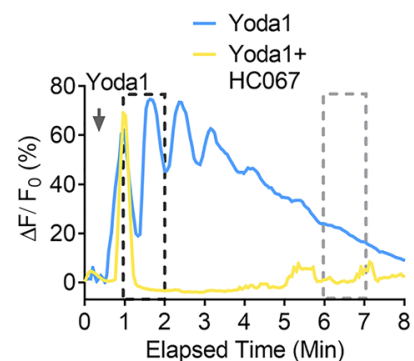

$\mathbf{K}$

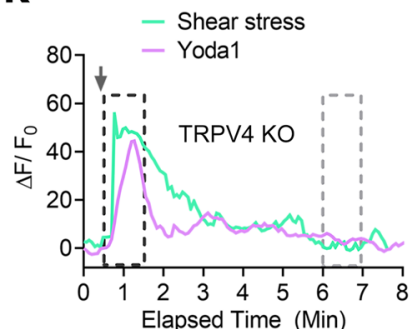

H Peak fluorescence Shear stress Shear stress $+\mathrm{HCO} 67$

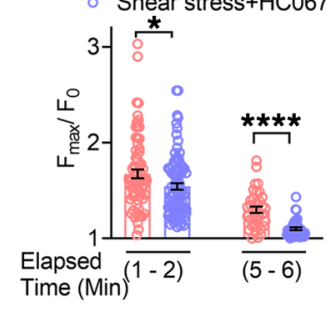

J

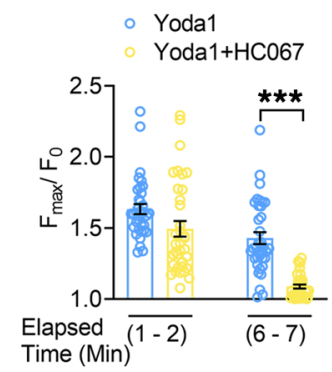

$\mathbf{L}$

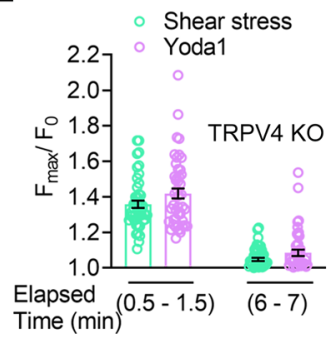

Figure 5. TRPV4 is expressed in pancreatic acini, and Piezo1 induces TRPV4 activation. (A) mRNA (fold expression) of TRPV4 and Piezo1 in pancreatic acini relative to the housekeeping gene actb ( $n=3-5$ experiments). (B) Immunostaining of mouse pancreatic acini with a TRPV4 antibody (red). Nuclei (blue) were stained with Nunc blue. Scale bar: $10 \mu \mathrm{m}$. (C and D) Effects of the TRPV4 agonist GSK101 (50 nM) and GSK101 + the TRPV4 antagonist HC067 $(100 \mathrm{nM})$ or RN1734 $(30 \mu \mathrm{M})$ on $\left[\mathrm{Ca}^{2+}\right]_{i}$ are shown. (C) A representative experiment shows the relative fluorescence intensity $\left(\Delta \mathrm{F} / \mathrm{F}_{0}\right)$ of calcium dye over time and (D) the average peak [Ca $\left.{ }^{2+}\right]$ intensity of pancreatic acini from 43 to 63 cells. (E and F) Arachidonic acid $(20 \mu \mathrm{M})$ and $5^{\prime}, 6^{\prime}-\mathrm{EET}(5 \mu \mathrm{M})$ increased $\left[\mathrm{Ca}^{2+}\right]_{\text {. }}$. The effects of $5^{\prime}, 6^{\prime}-\mathrm{EET}(5 \mu \mathrm{M})$ were blocked by HCO67 $(100 \mathrm{nM})$. (E) The relative fluorescence intensity of calcium dye and $(\mathbf{F})$ average peak $\left[\mathrm{Ca}^{2+}\right]_{\mathrm{i}}$ intensity of pancreatic acini from 40 to 54 cells are shown. (G, H, I, and J) The TRPV4 antagonist HC067 (1 $\mu \mathrm{M})$ blocked the effects of shear stress (12 dyne/ $\mathrm{cm}^{2}$ ) and Yoda1 $(25 \mu \mathrm{M})$ on the sustained $\left[\mathrm{Ca}^{2+}\right]$, responses. (G and I) Representative tracings of the relative fluorescence intensity of calcium dye over time with the different stimuli. ( $\mathbf{H}$ and $\mathbf{J}$ ) The average peak $\left[\mathrm{Ca}^{2+}\right]_{i}$ responses were calculated from 35 to 86 cells. (K and $\mathbf{L}$ ) The shear stress- and Yoda-induced sustained increases in $\left[\mathrm{Ca}^{2+}\right]_{i}$ were not seen in pancreatic acini isolated from TRPV4-KO mice. (K) Representative traces demonstrate the effects of shear stress or Yoda1 on $\left[\mathrm{Ca}^{2+}\right]$, and $(\mathbf{L})$ show the average peak $\left[\mathrm{Ca}^{2+}\right]$, intensity at different time intervals (from 45 to 47 cells). Statistical analyses were performed using Student's $t$ test. Data are shown as the mean \pm SEM. ${ }^{*} P \leq 0.05 ;{ }^{* * *} P \leq 0.001 ;{ }^{* * *} P \leq 0.0001$.

jected to fluid shear stress at 12 dyne $/ \mathrm{cm}^{2}$ for 30 seconds. Trypsin activity monitored by live-cell imaging was detected after 10 minutes of fluid shear stress and gradually increased for up to $50 \mathrm{~min}$ utes (Figure 4C). An increase in BZiPAR dye (trypsin activity) was

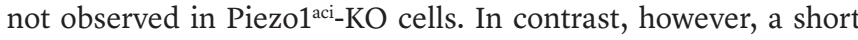
pulse of 5 seconds, instead of 30 seconds, at a force of 12 dyne $/ \mathrm{cm}^{2}$ did not trigger trypsin activation in WT acinar cells (Figure 4D). As we observed with mitochondrial depolarization, brief mechanical pushing of pancreatic acinar cells was not sufficient to activate trypsin (Supplemental Figure 4B).

Piezo1 mediates TRPV4 channel activation in pancreatic acini. Although Piezol is a fast inactivating channel, fluid shear stress and Yoda1 caused a sustained elevation in $\left[\mathrm{Ca}^{2+}\right]_{i}$, raising the possibility that other calcium entry channels or Piezo1-mediated downstream signaling pathways may exist. CRAC, which is expressed in pancreatic acinar cells (24), was one possibility; however, we observed that the CRAC inhibitor CM4620, which selectively inhibits the Orai channel, blocked the sustained elevation in $\left[\mathrm{Ca}^{2+}\right]_{i}$ produced by CCK, but not that induced by Yoda1 (Supplemental Figure 2). Therefore, it is unlikely that CRAC contributes to Piezo1-stimulated $\mathrm{Ca}^{2+}$ entry. Since TRPV4 indirectly senses fluid shear stress, we sought to determine whether TRPV4 was expressed in pancreatic acinar cells. As shown in Figure 5, A and B, TRPV4 mRNA and protein were detected by quantitative reverse transcriptase PCR (RT-qPCR) and immunostaining, respectively. Moreover, TRPV4 was highly expressed in both mouse and human pancreatic acini (Figure 5, A and B, and Supplemental Figure 5, A and C). Like mouse pancreatic acini, Piezo1 was also expressed in human pancreatic acini (Supplemental Figure 5B). In order to evaluate the function of TRPV4 in pancreatic acini, we used the TRPV4 channel agonist GSK10167790A (GSK101) and TRPV4 receptor antagonists HC067047 (HC067) and RN1734 (31, 32, 57). GSK101 (50 
A

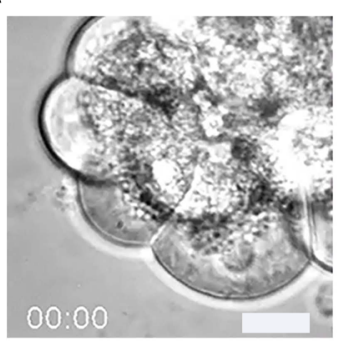

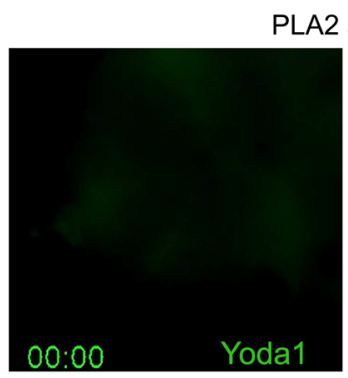

PLA2 activity

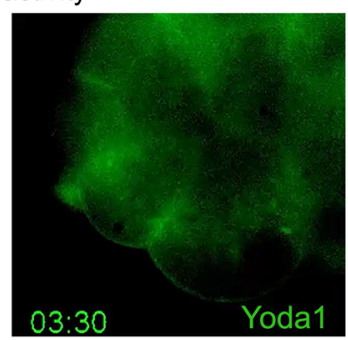

B

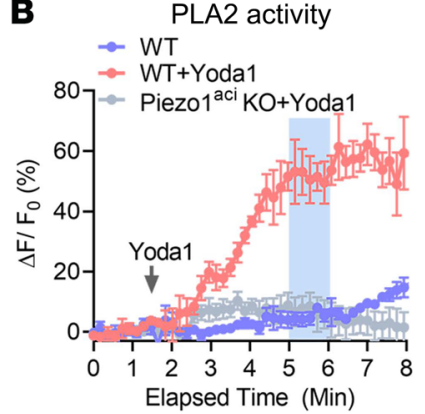

C PLA2 activity

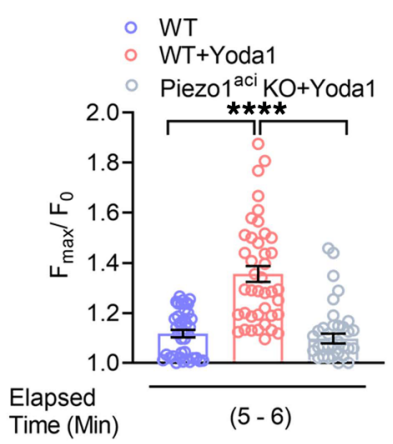

D

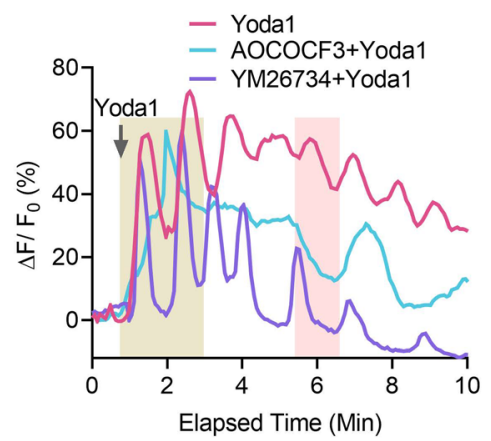

E
Peak calcium fluorescence

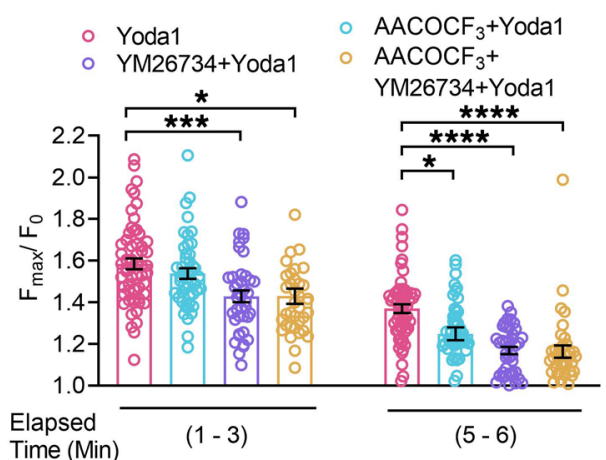

Figure 6. The Piezo1 agonist, Yoda1, induces activation of PLA2 activity. (A) Brightfield and fluorescence images of BODIPY FL $C_{11}-P C$ loaded pancreatic acini at time 0 and 3:30 minutes after Yoda1 $(25 \mu \mathrm{M})$ application. Scale bar: $10 \mu \mathrm{m}$. (B) Traces represent the live-cell PLA2 activity upon Yoda1 (25 M) application from 4 experiments. Representative tracings of acini from WT and Piezo1aci-KO mice are shown. The peak fluorescence intensity was calculated from the elapsed time at 5 to 6 minutes (blue bar). (C) Peak fluorescence intensity of BODIPY FL $C_{11}$-PC dye measured at an elapsed time of 5 to 6 minutes from 40 to 51 cells. (D and $\mathbf{E})$ The traces and graph show the effects of Yoda1 $(25 \mu \mathrm{M})$ on [Ca $\left.\mathrm{a}^{2+}\right]_{\mathrm{i}}$ in pancreatic acini with or without treatment with the cytoplasmic PLA2 blocker AACOCF3 $(30 \mu \mathrm{M})$ and secretory PLA2 blocker YM26734 (10 $\mu \mathrm{M})$. YM26734 and AACOCF3 were preincubated 10 minutes before application of Yoda1. The transient calcium peaks were measured from signals obtained between 1 to 3 minutes (yellow bar), and sustained calcium peaks were measured from signals from 5.5 to 6.5 minutes (pink bar). Data represent the averages of $36-58$ cells. Values were expressed as the mean \pm SEM, and mean differences between multiple groups were analyzed by 1-way ANOVA with Tukey's multiple comparison. ${ }^{*} P \leq 0.05$; ${ }^{* * *} P \leq 0.001 ;{ }^{* * * *} P \leq$ 0.0001 . Scale bar: $10 \mu \mathrm{m}$.

$\mathrm{nM})$ induced a significant increase in $\left[\mathrm{Ca}^{2+}\right]_{i}$, which was inhibited by both HC067 (100 nM) and RN1734 (30 $\mu \mathrm{M})$ (Figure 5, C and D).

To determine the mechanism for TRPV4 activation, we examined the PLA2 pathway by first testing the endogenous AA metabolite ligand 5',6'-EET. Similarly to the TRPV4 agonist GSK101, AA and $5^{\prime}, 6^{\prime}$-EET induced significant increases in $\left[\mathrm{Ca}^{2+}\right]_{\mathrm{i}}$ (Figure $5, \mathrm{E}$ and F) that were inhibited by the TRPV4 antagonist HCO67 (Figure 5, E and F). We next sought to determine whether the ability of shear stress to produce the sustained elevation in $\left[\mathrm{Ca}^{2+}\right]_{\mathrm{i}}$ was due to TRPV4 by measuring $\left[\mathrm{Ca}^{2+}\right]_{\mathrm{i}}$ following shear stress (12 dyne/ $\mathrm{cm}^{2}$ for 30 seconds) in the presence of the TRPV4 antagonist HC067. HC067 $(1 \mu \mathrm{M}$, the concentration used to completely inhibit TRPV4 activity) (31) blocked the sustained $\left[\mathrm{Ca}^{2+}\right]_{i}$ elevation, leaving only transient alterations in $\left[\mathrm{Ca}^{2+}\right]_{\mathrm{i}}$ (Figure $5, \mathrm{G}$ and $\mathrm{H}$ ). Like shear stress experiments, HC067 completely blocked the Yoda1-stimulated $(25 \mu \mathrm{M})$ sustained increase in $\left[\mathrm{Ca}^{2+}\right]_{\mathrm{i}}$ (Figure 5, I and J). Importantly, we discovered that neither Yoda1 nor fluid shear stress (12 dyne/ $\mathrm{cm}^{2}$ for 30 seconds) caused a sustained increase $\left[\mathrm{Ca}^{2+}\right]_{\mathrm{i}}$ in pancreatic acini isolated from TRPV4-KO mice (Figure 5, $\mathrm{K}$ and L). Together these findings indicate that Piezo1 directly senses fluid shear stress and initiates calcium influx. However, the activation of TRPV4 is responsible for the secondary sustained influx of calcium resulting in the sustained elevation in $\left[\mathrm{Ca}^{2+}\right]_{i}$.
Piezo1 elevates PLA2 activity. TRPV4 is activated by 5 ', $6^{\prime}$-EET generated through the PLA2-AA cytochrome P450 epoxygenase-dependent pathway (42). If this pathway is responsible for Piezo1-initated TRPV4 channel activation, then Yoda1 should be able to induce PLA2 activation. To test this hypothesis, acinar cells were loaded with the fluorogenic PLA2 substrate 1, 2-Bis (4, 4-difluoro-5, 7-dimethyl-4-Bora-3a, 4a-diaza-s-indacene-3-undecanoyl)-Sn-glycero-3-phosphocholine (Bis-BODIPY FL C ${ }_{11}^{-}$ PC). Prior to Yoda1 stimulation, initial Bis-BODIPY FL $\mathrm{C}_{11}-\mathrm{PC}$ loaded pancreatic acini exhibited only faint fluorescence. However, application of Yoda1 markedly increased the intensity of the fluorogenic PLA2 substrate, indicating that Piezo1 is able to induce PLA2 activity (Figure 6, A and B, and Supplemental Video 8). The effects of Yoda1 were observed within 30 seconds of application and reached a plateau after 4 minutes (Figure $6 \mathrm{~B})$. Yoda1 did not stimulate PLA2 activation in pancreatic acini from Piezo1 ${ }^{\text {aci }}$-KO mice (Figure 6, B and C). To confirm that the increase in PLA2 activity was responsible for the Piezo1-induced sustained $\left[\mathrm{Ca}^{2+}\right]_{\mathrm{i}}$ levels, we tested the secretory and cytosolic PLA2 inhibitors YM26734 and AACOCF3, respectively $(58,59)$. Together, YM26734 and AACOCF3 blocked the Yoda1-induced sustained $\left[\mathrm{Ca}^{2+}\right]_{\mathrm{i}}$ elevation, indicating that Piezo1 induced PLA2, which was responsible for the subsequent 
A
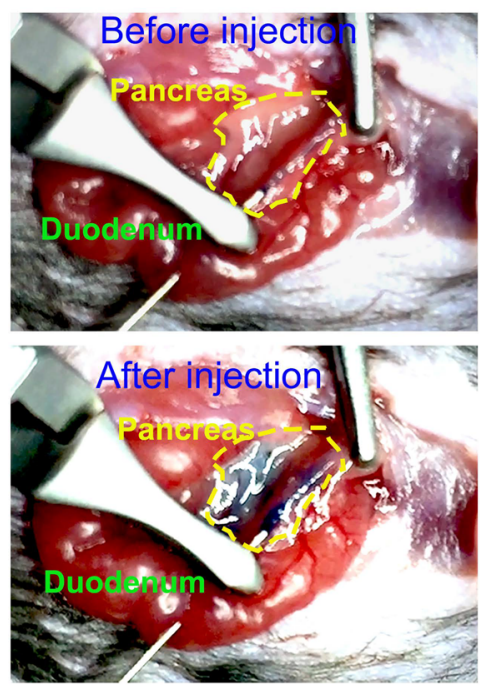

B

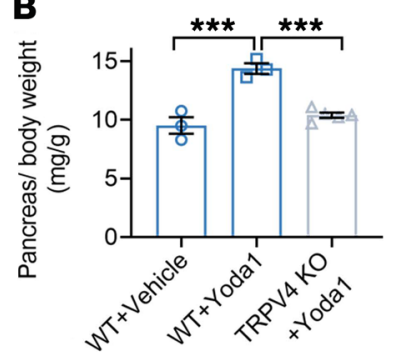

D

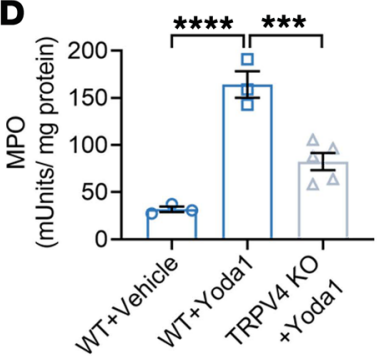

C

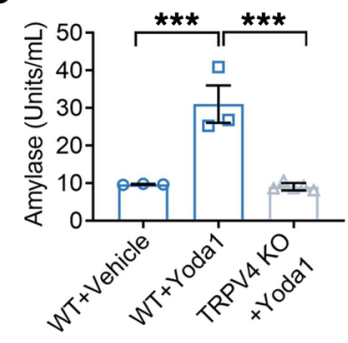

E

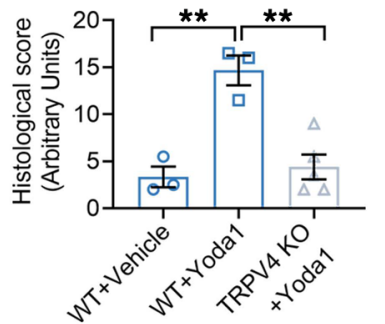

$\mathbf{F}$

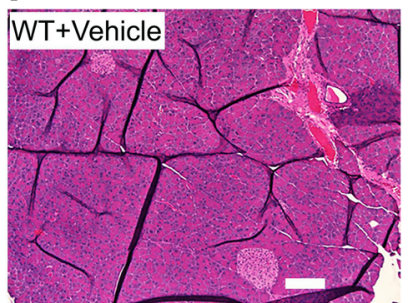

G

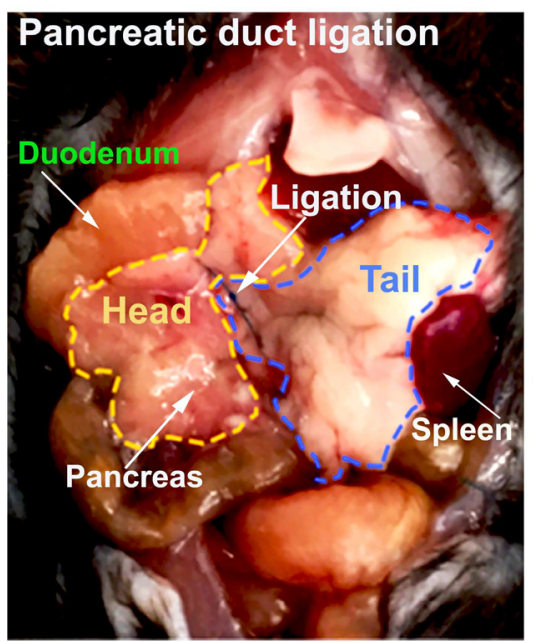

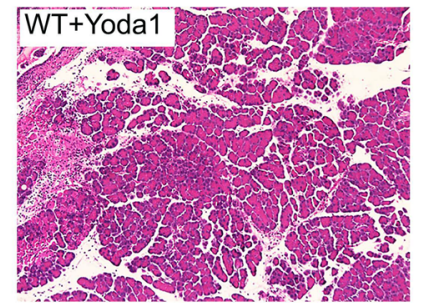

H

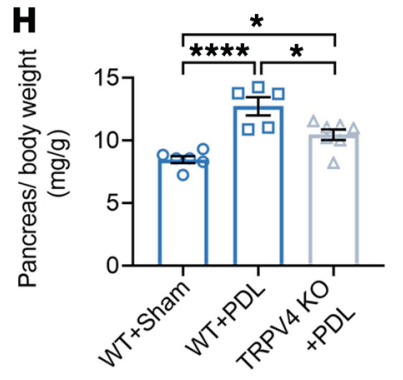

J

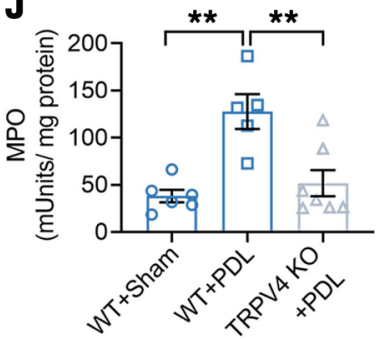

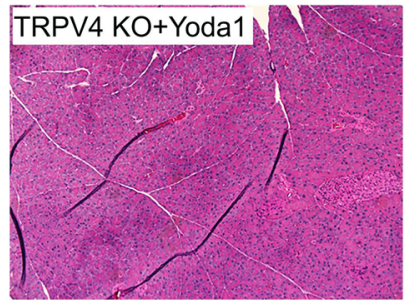

I

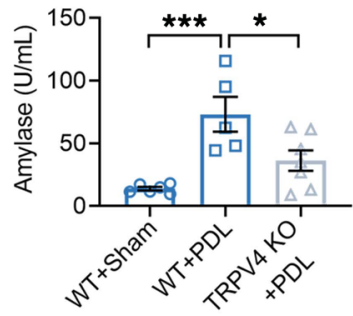

K

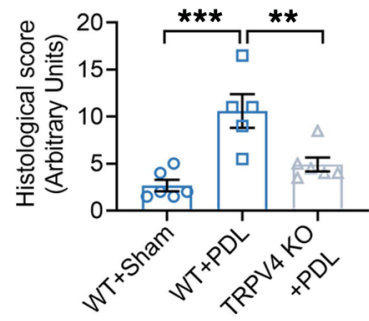

L

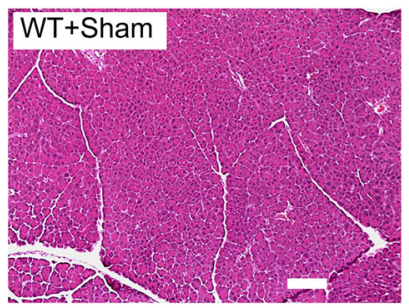

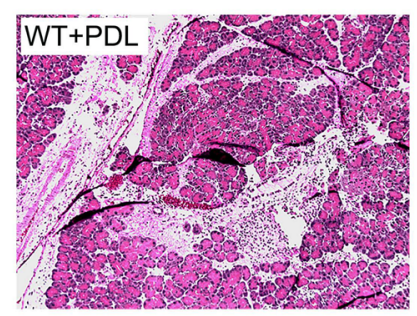

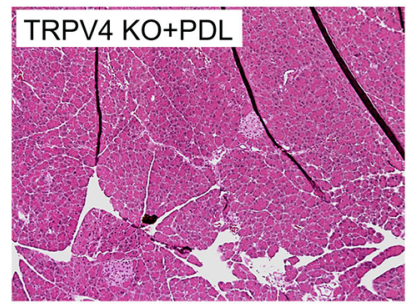

Figure 7. TRPV4-KO mice are protected against Yoda1- and pancreatic pressure-induced pancreatitis. (A) Photograph of the surgical approach used for injection of Yoda1 into the pancreatic duct is shown. Methylene blue dye was mixed with the injected solution to aid with pancreatic duct visualization. A total of $50 \mu \mathrm{L}$ was injected over 10 minutes. The amount of Yoda1 injected into the pancreatic duct was $0.4 \mathrm{mg} / \mathrm{kg}$. The boundary of the mouse pancreas is marked with yellow before and after Yoda1 infusion. After injection, methylene blue in the solution made the pancreas blue in color. Pancreatitis parameters included edema (B), serum amylase (C), tissue MPO (D), and pancreatic histology score (E) in vehicle- and Yoda1-treated WT and TRPV4-KO mice ( $n=$ 3-5). (F) Representative H\&Estained images of the midregion of pancreas are shown. Scale bar: $100 \mu \mathrm{m}$. (G) Photograph of the partial PDL procedure is shown. Head and tail regions of pancreas are outlined in yellow and blue, respectively. PDLinduced pancreatitis parameters included edema (H), serum amylase (I), pancreatic MPO (J), and pancreas histology scores (K) of the sham and PDL WT and TRPV4-KO mice $(n=5-7)$. (L) Representative H\&E images of the midregion of the pancreas are shown. Scale bar: $100 \mu \mathrm{m}$. Statistical analyses were performed using 1-way ANOVA with Tukey's multiple comparison. Data are shown as mean \pm SEM ${ }^{*} P \leq 0.05 ;{ }^{* *} P \leq 0.01 ;{ }^{* * *} P \leq$ $0.001 ;{ }^{* * *} P \leq 0.0001$ 
$\left[\mathrm{Ca}^{2+}\right]_{\mathrm{i}}$ elevation (Figure 6, D and E). However, only the secretory PLA2 inhibitor effect was substantial and capable of blocking the sustained $\left[\mathrm{Ca}^{2+}\right]_{\mathrm{i}}$ elevation (Figure 6, D and E). PKA and PKC inhibitors did not significantly affect Yoda-stimulated $\left[\mathrm{Ca}^{2+}\right]_{\mathrm{i}}$ (Supplemental Figure 6).

TRPV4-KO mice are protected against pressure-induced pancreatitis. Sustained elevations in $\left[\mathrm{Ca}^{2+}\right]_{\mathrm{i}}$ are sufficient to cause pancreatitis (60). Having demonstrated that Piezo1-initiated, sustained $\left[\mathrm{Ca}^{2+}\right]_{\mathrm{i}}$ elevation requires TRPV4 activation, we proposed that TRPV4-KO mice could be protected from Piezo1-mediated pancreatitis. In order to selectively stimulate Piezo1 in the pancreas, Yoda1 $(0.4 \mathrm{mg} / \mathrm{kg})$ was infused into the pancreatic duct at a rate of $5 \mu \mathrm{L} / \mathrm{min}$ (Figure 7A). This rate of infusion did not exceed the pancreatic duct pressure of $11 \mathrm{mmHg}$ and is considered a low-pressure condition (1) that alone does not cause pancreatitis. As shown in Figure 7, B-F, Yoda1 infusion increased all pancreatitis parameters measured (pancreatic edema, serum amylase, pancreatic myeloperoxidase [MPO], and histological scoring) in WT mice. In contrast, the same dose of Yoda1 did not cause pancreatitis in TRPV4KO mice (Figure 7, B-F).

Having demonstrated that Piezo1 and CCK increase $\left[\mathrm{Ca}^{2+}\right]_{i}$ through separate mechanisms, we proposed that CCK would not trigger TRPV4 channel opening. In support of this idea, we found that the TRPV4 channel blocker HC067 did not affect the CCK-stimulated $(1 \mathrm{nM})$ peak or sustained $\left[\mathrm{Ca}^{2+}\right]_{\mathrm{i}}$ changes (Supplemental Figure 7, A-C). It follows then that if CCK does not stimulate TRPV4 opening in acini, TRPV4-KO mice would not be protected from caerulein-induced pancreatitis. As expected, caerulein-induced pancreatitis was similar in both WT and TRPV4-KO mice (Supplemental Figure 7, D-H).

Piezo1 is responsible for pressure-induced pancreatitis (1). Experimentally, Piezo1 ${ }^{\text {aci }}$-KO mice were protected against pancreatitis caused by high intrapancreatic duct pressure (1). If the pathological effects of Piezo1 activation are due to the downstream activation of TRPV4, we would expect that mice lacking TRPV4 would be protected against pressure-induced pancreatitis. We induced pancreatitis by ligating the tail region of the pancreas up to 24 hours (Figure $7 G$ ). In WT mice, pancreatic duct ligation (PDL) caused acute tissue injury that was reflected by an increase in all pancreatitis parameters. In contrast, TRPV4-KO mice were protected against duct ligation-induced pancreatitis (Figure 7, H-L). These findings confirm that TRPV4 plays a key role in pressure-induced pancreatitis.

\section{Discussion}

Acinar cells make up $90 \%$ of the pancreas and are notable for their abundant digestive enzymes that render the gland highly susceptible to pancreatitis should enzymes become prematurely activated following organ damage. Intracellular enzyme activation is a hallmark of pancreatitis and is believed to play a central role in disease pathogenesis. Recently, we demonstrated that pressure activation of Piezo1 channels in pancreatic acinar cells is responsible for pressure-induced pancreatitis (1). Prior to the identification of Piezo1 in acinar cells, it was not known how the pancreas senses pressure, although a number of pathological situations indicate that the gland is exquisitely sensitive to pressure-induced injury. For example, intrapancreatic duct pressure is increased by occlusion of the pancreatic duct (4-6) and this is thought to be a key factor in the development of gallstone-induced pancreatitis, which is one of the major causes of acute pancreatitis in humans. In this disease, impaction of a gallstone in the ampulla of Vater causes an abrupt increase in pancreatic duct pressure, leading to pancreatitis (61). In addition, mechanical force on the pancreas during trauma or pancreatic surgery may cause pancreatitis $(7,9)$. Finally, injection of fluid into the pancreatic duct at high pressure during ERCP is a well-known cause of pancreatitis $(7,62,63)$. In each of these causes of pancreatitis, abnormal pressure on the pancreas has the potential to activate Piezo1. However, Piezo1 is also expressed in other tissues, where it mediates several physiological processes, such as micturition, endothelial shear stress, embryogenesis, and vascular development $(12,13,16,17,64)$. The question, therefore, arises of how activation of the Piezo1 channel leads to the pathological process of pancreatitis. In general, under normal physiological conditions, activation of Piezo1 channels is tightly regulated. Piezo1 channel function and transduction properties vary with stimulus frequency, waveform, and duration (65). We propose that overactivation of Piezo1 by persistent pressure or mechanical force in acinar cells produces downstream signaling events that disrupt normal cellular homeostasis, resulting in premature enzyme activation and ultimately pancreatitis. In the pancreas, disruption of calcium homeostasis is detrimental and leads to pancreatitis $(23,66)$.

Here, we demonstrate that either chemical (Yoda1) or physical (shear stress) activation of Piezo1 induced $\left[\mathrm{Ca}^{2+}\right]_{\mathrm{i}}$ overload, caused mitochondrial dysfunction, and led to intrapancreatic trypsinogen activation. Persistent application of the Piezo1 agonist Yoda1 at doses of $25 \mathrm{M}$ and $50 \mu \mathrm{M}$ produced a sustained increase in $\left[\mathrm{Ca}^{2+}\right]_{\mathrm{i}}$ in pancreatic acini. A similar increase in $\left[\mathrm{Ca}^{2+}\right]_{i}$ was seen when cells were subjected to fluid shear stress of more than 12 dyne/ $\mathrm{cm}^{2}$ for 30 seconds. However, mechanical pushing of the acinar cell surface up to $5 \mu \mathrm{m}$ for 1 second caused only a transient rise in $\left[\mathrm{Ca}^{2+}\right]_{\mathrm{i}}$ and did not induce trypsinogen activation. Similarly, fluid shear stress at low pressure $\left(4 \mathrm{dyne} / \mathrm{cm}^{2}\right)$ or for a short duration (1 or 5 seconds) did not induce these changes. It has been established previously that a sustained elevation in $\left[\mathrm{Ca}^{2+}\right]_{\mathrm{i}}$ in acinar cells is a prime cause of pancreatic injury $(23,66)$. Elevated $\left[\mathrm{Ca}^{2+}\right]_{i}$ in pancreatic acinar cells produced by persistent Yoda1 exposure resembles that produced by supraphysiological doses of CCK, a wellknown agent for inducing experimental pancreatitis (66). Thus, it seems reasonable to attribute the ability of Yoda1 to induce pancreatitis to its prolonged effects on $\left[\mathrm{Ca}^{2+}\right]_{\mathrm{i}}$. It is possible that brief push stimulation activates a subset of Piezo1 and chemical activation acts on all channels, which mimics the prolonged high shear stress-mediated pathological effects.

We observed that removal of external $\mathrm{Ca}^{2+}$ abolished the Piezo1-mediated increase in $\left[\mathrm{Ca}^{2+}\right]_{i}$ and preincubating cells with the cell-permeable calcium chelator (BAPTA-AM) blocked the sustained increase in $\left[\mathrm{Ca}^{2+}\right]_{i}$. Thus, it is possible that not only is external $\mathrm{Ca}^{2+}$ necessary for the Piezo1-mediated increase in $\left[\mathrm{Ca}^{2+}\right]_{\mathrm{i}}$, but also this increase is required for the opening of a $\mathrm{Ca}^{2+}$ entry pathway that contributes to the sustained elevation in $\left[\mathrm{Ca}^{2+}\right]_{\mathrm{i}}$. This is consistent with the observation that preincubating cells with BAPTA-AM protected acini from Piezo1-mediated cellular injury. Notably, Piezo1-mediated stimulation of $\left[\mathrm{Ca}^{2+}\right]_{i}$ differs from 
stimulation through the the CCK-mediated pathway. The Piezo1 pathway requires external $\mathrm{Ca}^{2+}$ to initiate the process, whereas CCK stimulation begins via the release of $\mathrm{Ca}^{2+}$ from intracellular ER stores $(23,67)$.

Under physiological conditions, the pancreatic secretagogues acetylcholine and CCK stimulate $\left[\mathrm{Ca}^{2+}\right]_{\mathrm{i}}$ in a spatiotemporal manner that is required for ATP production, initiation of exocytosis, and nuclear signaling processes (68). However, supraphysiological CCK stimulation and excess ethanol, bile, and toxins induce a sustained elevation in $\left[\mathrm{Ca}^{2+}\right]_{\mathrm{i}}$ in pancreatic acinar cells that cause acute pancreatitis $(28,69-72)$. This sustained elevation in $\left[\mathrm{Ca}^{2+}\right]_{i}$ mediates mitochondrial dysfunction, premature zymogen activation, vacuolization, and necrosis (73). We observed that both Yoda1 and fluid shear stress induced mitochondrial depolarization and trypsin activation in pancreatic acinar cells. Transient depolarization of mitochondria following a rise in $\left[\mathrm{Ca}^{2+}\right]_{\mathrm{i}}$ is associated with normal cellular ATP production. However, $\left[\mathrm{Ca}^{2+}\right]_{i}$ overload in acinar cells induced by bile and ethanol $(27,28,71,74)$ opens the MPTP, collapses the mitochondrial membrane potential ( $\psi \mathrm{m}$ ) required for ATP synthesis (75-77), and ultimately results in cell death. By chelating intracellular-free $\mathrm{Ca}^{2+}$, we prevented Yoda1-induced mitochondrial depolarization and cell death. It has been observed previously that intracellular calcium chelation prevents zymogen activation $(52,78)$ and protects against acute pancreatitis in vivo (79). We observed that Piezo1-mediated mitochondrial depolarization preceded trypsin activation following either Yoda1 treatment or shear stress and is ultimately responsible for pressure-induced pancreatitis.

Piezo1 is a fast inactivating channel with single-channel conductance of approximately $22 \mathrm{pS}$ (inward current), which is lower than the TRPV4 ion channel of approximately $60 \mathrm{pS}(10,80)$. Piezo1 inactivation kinetics are independent of stimulus intensity (10). If no other type of channel is present in the cell except Piezo1, the calcium rise will be transient rather than sustained due to fast inactivation kinetics. This suggests that the Piezo1-induced sustained elevation in $\left[\mathrm{Ca}^{2+}\right]_{\mathrm{i}}$ produced by Yoda1 or shear stress requires an extra calcium entry pathway. We, therefore, sought other potential channels that could be linked to Piezo1 activity and discovered that TRPV4 is expressed in both mouse and human pancreatic acini.

Initially, we thought that mechanical activation of Piezo1 and TRPV4 were independent processes. Even though activation of Piezo1 could produce a transient increase in $\left[\mathrm{Ca}^{2+}\right]_{\mathrm{i}}$ and TRPV4 could produce more sustained elevation of $\left[\mathrm{Ca}^{2+}\right]_{\mathrm{i}}$ by virtue of its slow inactivation kinetics and considerably higher single channel conductance, it was not clear whether the 2 processes were linked. Remarkably, the TRPV4 antagonist HC067 completely blocked the sustained phase of calcium elevation induced by Yoda1 and shear stress. This provided the hint that Piezo1 regulates TRPV4 channel activation. The results were confirmed in experiments from TRPV4-KO mice when both Yoda1 and prolonged shear stress produced only transient elevation in $\left[\mathrm{Ca}^{2+}\right]_{i}$. Low shear stress for 30 seconds and high shear stress for 5 seconds were not sufficient to induce a sustained calcium rise and did not activate TRPV4. The reason could be that the brief force caused only a subset of Piezo1 channel openings and was insufficient to activate PLA2. In the cell, PLA2 is activated upon binding to calcium ions that accelerate enzyme activity, which initiates the arachidonic pathway and TRPV4 channel activation. In mouse pancreatic acinar cells, the Piezo1 agonist Yoda1 increased PLA2 activity and caused a sustained elevation in $\left[\mathrm{Ca}^{2+}\right]_{i}$, an effect that was inhibited by a PLA2 inhibitor. Various reports indicate that TRPV4 is sensitive to mechanical stimuli, such as osmotic pressure, shear stress, and mechanical stretching (31-37). However, it was unclear how mechanical stimuli actually activate the TRPV4 channel. The current findings indicate that Piezo1 stimulation of PLA2 and subsequent activation of TRPV 4 could be a mechanism by which TRPV4 channels respond to mechanical force.

Supramaximal doses of CCK secretagogue block apical secretion and cause intracellular vacuolization, enzyme activation, and enzyme release from the basolateral surface of the cell $(81,82)$. In the present study, we observed that Yoda1 induced the release of vesicles from the basolateral surface, indicating a possible pathological situation.

We did not observe any significant effects of PKA and PKC inhibitors on Yoda1-mediated TRPV4 activation. In other cells, such as HEK293 cells or human coronary artery endothelial cells, PKA and PKC can directly phosphorylate TRPV4 and modify channel activity. Our findings suggest that this pathway is not required for channel activation.

The current study demonstrates that Piezo1 initiates the pressure-induced calcium signal, causing TRPV4 activation, but the pathological events that occur in the acinar cell require TRPV4-mediated calcium influx, which is responsible for the sustained phase of calcium elevation that leads to pancreatitis. Consistent with this pathological sequence of events, TRPV4-KO mice were protected from Yoda1-induced pancreatitis.

To mimic pancreatitis caused by pancreatic duct obstruction (e.g., gallstones), we used a mouse model of pancreatitis by ligating the tail region of the pancreas for 24 hours (83). In this model, we observed that TRPV4-KO mice were substantially protected. Hence, a TRPV 4 channel blocker could be a possible treatment for pancreatitis where pressure is encountered.

Our findings suggest that activation of Piezo1 in the absence of TRPV4 is not sufficient for inducing pathological calcium signaling. However, when coexpressed and linked by intracellular signaling pathways, TRPV4 may appear to be pressure sensitive. For example, Piezo1 and TRPV4 channels are expressed in endothelial cells, and previous reports have indicated that high pulmonary venous pressure induces $\mathrm{Ca}^{2+}$ influx into endothelial cells via TRPV4 channels, resulting in increased vascular permeability, which is a major cause of mortality in heart failure patients (33). Although unrecognized at the time, this process may be linked to Piezo1, which appeared to sense high vascular pressures at the lung endothelial surface and account for vascular hyperpermeability and pulmonary edema (30). Thus, it appears that both Piezo1 and TRPV4 are responsible for this vascular hyperpermeability, and our findings suggest that they may be linked. We hypothesize that Piezo1 sensing of high vascular pressure initiates a $\mathrm{Ca}^{2+}$-signaling pathway that triggers the activation of TRPV4. TRPV4 activation would then cause a secondary, sustained $\mathrm{Ca}^{2+}$ influx that would lead to vascular hyperpermeability. The extent of TRPV4-induced $\mathrm{Ca}^{2+}$ entry would be influenced by other factors, including the level of TRPV4 expression, the degree and duration of pressure, and, if identical to the pancreas, 
the appropriate level of PLA2 activity. Thus, our findings may represent a more generalized process in which TRPV4 converts Piezo1 pressure sensing into a pathological event.

\section{Methods}

Animals. Targeted deletion of Piezo1 in pancreatic acinar cells was accomplished as follows. Piezo $1^{f / / f l}$ mice were a gift from A. Patapoutian (Department of Neuroscience, Scripps Research, La Jolla, California,

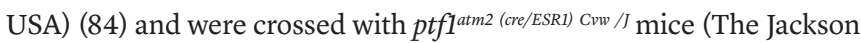

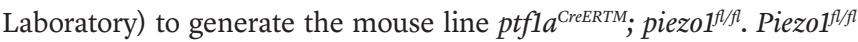
mice were used as WT, and $p t f 1 a^{\text {CreERTM }}$; piezo $1^{f / f l}$ mice at the age of 5 to 7 weeks were subjected to $1 \mathrm{mg}$ of tamoxifen (MilliporeSigma, T5648) injected intraperitoneally for 5 consecutive days. After tamoxifen induction, the $p t f 1 a^{\text {CreERTM}}$; piezo $1^{f / / f l}$ mice expressed a truncated Piezo1 specific to pancreatic acinar cells and are referred to as Piezo $1^{\text {aci-KO }}$ mice. Eight days after the last tamoxifen injection, mice were used in experiments, and each time a small piece of pancreas was used for genotyping. Mice (both male and female) aged 7 to 12 weeks were used in the experiments. Piezo $1^{f / f l}$ mice (8 to 12 weeks of age) on a C57BL/6J background (1) were used as WT mice, and ptfla $a^{\text {CreERTM }}$; piezo $1^{f l / f l}$ mice after tamoxifen injection were used as Piezo1 ${ }^{\text {aci }}$-KO mice for experiments with the Piezo1 agonist, Yoda1, and shear stress. C57BL/6J mice ( 8 to 14 weeks old) were used as WT mice, and mice with the trpv $4^{-/}$ gene deletion (referred to as TRPV4-KO), provided in-house (85), backcrossed on a C57BL/6J background, were used for both Yoda1 and partial duct ligation-mediated pancreatitis experiments. For in vivo experiments with Yoda1, WT mice were also injected with tamoxifen. Mice were housed under standard 12-hour light/12-hour dark periods.

Pancreatic acini and acinar cell preparations. Mouse pancreatic acini were isolated using the standard collagenase digestion protocol, as previously described $(1,86,87)$. Isolated acini were plated on a thin-layered, Matrigel-coated, glass-bottom, culture plate (MatTek, P35G-0-14-C). Freshly isolated acini were used in each experiment.

Shear stress assays. Parallel-plate fluid flow chambers ( $\mu$-Slide I 0.4 Luer, and $\mu$-Slide I 0.2 Luer from Ibidi $\mathrm{GmbH}$ ) were used to measure the shear stress-induced changes in intracellular calcium, mitochondrial depolarization, and trypsin activation (13). The constant flow rate with shear stress $(\tau)$ was determined as follows: $\tau=\eta \times 104.7 .6 \varphi$ for $\mu$-Slide I 0.4 Luer and $\tau=\eta \times 330.4 \varphi$ for $\mu$-Slide I 0.2 Luer, where $\eta=$ viscosity of the medium and $\varphi=$ flow rate (according to the manufacturer's instructions, Ibidi).

Mechanical pushing. A borosilicate glass pipette (Sutter Instrument) was pulled using a pipette puller P-87 (Sutter Instruments) and made blunt with an MF-900 Microforge (Narishige). Acini were pushed once with a 2 - to $3-\mu \mathrm{m}$ tip blunt pipette to $5 \mu \mathrm{m}$ for 1 second using a micromanipulator (World Precision Instruments).

Calcium imaging. Calcium imaging in pancreatic acini was performed as described previously (1). The chemicals used in calcium imaging experiments included the following: Yoda1 (Tocris; 5586), GsMTx4 (Abcam; ab141871), 5',6'-EET (Santa Cruz Biotechnology; sc-221066), AA (MilliporeSigma; A3611), HC067047 (Tocris; 4100), GSK1016790A (MilliporeSigma; G0798), RN1734 (Tocris; 3746), AACOCF3 (Tocris; 1462), YM26734 (Tocris; 2522), GF109203X (Tocris; 0741), and CCK8 (Sigma-Aldrich).

Mitochondrial depolarization. Live-cell mitochondrial depolarization was analyzed according to a previously described protocol (52) using the mitochondrial labeling dye TMRE. Isolated acini were plated on a thin-layered, Matrigel-coated, glass-bottom culture plate with DMEM/ F12 and $10 \%$ FBS media and placed in a $\mathrm{CO}_{2}$ incubator for 1 hour at $37^{\circ} \mathrm{C}$. After 1 hour, the acini were incubated with TMRE $(200 \mathrm{nM})$ in the bath buffer containing $140 \mathrm{mM} \mathrm{NaCl}, 4.7 \mathrm{mM} \mathrm{KCl}, 2.0 \mathrm{CaCl}_{2}, 1 \mathrm{mM} \mathrm{MgCl}_{2}, 10$ mM HEPES, and $10 \mathrm{mM}$ glucose ( $\mathrm{pH}$ adjusted to 7.4 with $\mathrm{NaOH}$ ) for 30 minutes. The TMRE dye was washed and replaced with fresh bath buffer. The images were captured with a Zeiss Axio observer Z1 microscope with MetaMorph software (Molecular Devices) at intervals of $600 \mathrm{~ms}$. TMRE was excited at 540-600 $\mathrm{nm}$ and emission collected at 585-675 $\mathrm{nm}$. Carbonyl cyanide 4-(trifluoromethoxy) phenylhydrazone (FCCP), which uncouples the oxidative phosphorylation process and depolarizes mitochondria, was used as a positive control $(52,53)$. A maximum of 2 mitochondrial puncta were taken per acinar cell (53).

Trypsinogen activation. To visualize Piezo1-induced trypsinogen activation, acini were loaded with active trypsin enzyme substrate BZiPAR $(10 \mu \mathrm{M})(52,55)$. Na-HEPES buffer with $2 \mathrm{mM} \mathrm{Ca}^{2+}$ was used during imaging. A Zeiss Axio observer Z1 with a high-sensitivity EMCCD camera with a $\times 40 / 0.75$ EC-Plan-NeoFluar DIC objective was used to capture the $Z$-stack images with stack thickness of $3 \mu \mathrm{m}$ at an interval of 12 seconds. The BZiPAR fluorescent wavelength Ex/Em was 498/521 nm. BZiPAR was excited at 470-510 nm and emission collected at $495-550 \mathrm{~nm}$. The captured images were analyzed with MetaMorph software (Molecular Devices).

Cell death assays. The viability of pancreatic acini with Yoda1 and CCK treatments was analyzed using the Live/Dead Cell Imaging Kit (Thermo Fisher Scientific, catalog R37601) (88) or the LDH Release Assay Kit (Promega, catalog G1780) (1).

PLA2 activity. The fluorogenic PLA2 substrate (Bis-BODIPY FL $\mathrm{C}_{11}{ }^{-}$ PC) (Thermo Fisher Scientific; B7701) was used to monitor PLA2 activity in living cells. The pancreatic acini were incubated with Bis-BODIPY FL C ${ }_{11}-\mathrm{PC}$ in HBSS buffer with $2 \mathrm{mM} \mathrm{Ca}^{2+}$ for 30 minutes. HBSS buffer with $2 \mathrm{mM} \mathrm{Ca}^{2+}$ was used during imaging. A Zeiss Axio observer Z1 with a high-sensitivity EMCCD camera with a $\times 40 / 0.75$ EC-Plan-NeoFluar DIC objective was used to capture the $Z$-stack images with stack thickness of $4 \mu \mathrm{m}$ at an interval of 10 seconds with $480 \mathrm{~nm}$ excitation and 525-nm emission filter. The captured images were analyzed with MetaMorph software (Molecular Devices).

Immunostaining. Pancreatic acini were incubated with a rabbit anti-TRPV4 antiserum (Alomone; ACC-034; 1:250) or with a rabbit anti-Piezo1 antiserum (Alomone; APC-087; 1:300) overnight at $2^{\circ} \mathrm{C}-8^{\circ} \mathrm{C}$. The signals from TRPV 4 and Piezo1 immunostaining were amplified by the tyramide signal amplification method using a kit (Life Technologies; T20924) following the manufacturer's instructions. The nuclei were stained with Nunc blue (Invitrogen; R376060). All staining images were taken with a Zeiss Axio observer Z1 with a $\times 20$ objective or a $\times 63$ oil-immersion objective.

In vivo pancreatitis models. Laparotomy surgery was performed both in pancreatic partial duct ligation- and retrograde pancreatic duct infusion-mediated pancreatitis experiments as previously described (1). Yoda1 at a dose of $0.4 \mathrm{mg} / \mathrm{kg}$ in $50 \mu \mathrm{L} 1.1 \%$ dimethylsulfoxide, $4.8 \%$ ethanol, and $94.1 \%$ buffered saline was injected as previously described (1). In Yoda1-mediated pancreatitis, the midportion of the pancreas (about 100-125 mg) was carefully excised and used for biochemical assays and histological staining. Histological scoring was evaluated from the entire section. Partial PDL was performed as previously described $(89,90)$. Using a stereo microscope, the tail region of the pancreas was visualized, and the main pancreatic duct was ligat- 
ed carefully with 6-0 Prolene suture without damaging the left portal vein that separates the splenic lobe and gastroduodenal part of the pancreas. Any mice suffering damage to any underlying blood vessels were excluded from the experiment. The pancreas was examined 24 hours after ligation. A 100- to 125-g portion of the tail region of the pancreas was used for biochemical assays and histological staining. Mice were subjected to caerulein-induced pancreatitis by intraperitoneal injection of caerulein $(50 \mu \mathrm{g} / \mathrm{kg})$ (Tocris; 6264) every hour for a total of 6 injections as previously described (85).

Blood amylase assay. Serum amylase concentration was measured by a colorimetric method after reaction with substrate using the Phadebas Amylase Test Tablet (Magle Life Sciences) and the Tecan-infinite M200 Pro plate reader (1).

MPO assay. MPO was measured using previously described methods with modifications (1). To calculate the $\mathrm{mU}$ of $\mathrm{MPO} / \mathrm{mg}$ of protein, the protein concentrations of the supernatants were measured using the Micro BCA Protein Assay Kit (Thermo Fisher Scientific; 23235).

$H \& E$ staining. In Yoda1-mediated pancreatitis, the body region of the pancreas was used for H\&E staining (1), and in partial duct ligation-mediated pancreatitis, the tail region of the pancreas was used. The histological score was calculated from the pathological parameters, e.g., tissue edema, neutrophil infiltration, necrosis, and hemorrhage, with a minimum to severe scoring range of $0-3,0-3$, $0-7$, and $0-7$, respectively (91). The scores from all parameters were added to obtain a total histological score.

$R T-P C R$. RNAs were isolated using the RiboPure Kit (Invitrogen; AM1924), followed by DNase I digestion (Invitrogen; AM1906) (1).

Statistics. Results are expressed as mean \pm SEM. Mean differences between 2 groups were analyzed by 2 -tailed Student's $t$ test, and mean differences between multiple groups were analyzed by 1-way ANOVA with Tukey's multiple comparison posttest (GraphPad Prism 8). P values of less than 0.05 were considered significant.

Study approval. Experimental protocols and studies were performed with approval from the Institutional Animal Care and Use Committee of Duke University.

\section{Author contributions}

RAL, SMS, and JMJR designed experiments. SMS, JMJR, and RAS performed experiments and analyzed data. SMS and RAL wrote the manuscript. SJP and SRV provided critical review of the data and advice throughout the research. WL reviewed the manuscript, offered advice, and provided TRPV4-KO mice. RAL supervised the project and provided funding for the project. All authors reviewed the manuscript.

\section{Acknowledgments}

This work was supported by NIH grants DK064213 and DK120555 and the Department of Veterans Affairs. The authors wish to thank Jorg Grandl for helpful advice and for reviewing the manuscript. We acknowledge the generous assistance of Yasheng Gao from the Duke University Light Microscopy Core. The authors also acknowledge support from the Duke Comprehensive Cancer Center and the Duke Transgenic Core Facilities.

Address correspondence to: Rodger A. Liddle, Box 103859, 1033A Genome Science Research Building 1, 905 LaSalle Street, Duke University Medical Center, Durham, North Carolina 27710, USA. Phone: 919.681.6380; Email: rodger.liddle@duke.edu.
1. Romac JM, Shahid RA, Swain SM, Vigna SR, Liddle RA. Piezo1 is a mechanically activated ion channel and mediates pressure induced pancreatitis. Nat Commun. 2018;9(1):1715.

2. Yamamoto M, Otani M, Otsuki M. A new model of chronic pancreatitis in rats. Am J Physiol Gastrointest Liver Physiol. 2006;291(4):G700-G708.

3. Watanabe $S$, et al. Pressure activates rat pancreatic stellate cells. Am J Physiol Gastrointest Liver Physiol. 2004;287(6):G1175-G1181.

4. Opie EL, Meakins JC. Data concerning the etiology and pathology of hemorrhagic necrosis of the pancreas (acute hemorrhagic pancreatitis). J Exp Med.1909;11(4):561-578.

5. Lerch MM, Saluja AK, Rünzi M, Dawra R, Saluja M, Steer ML. Pancreatic duct obstruction triggers acute necrotizing pancreatitis in the opossum. Gastroenterology. 1993;104(3):853-861.

6. Gukovskaya AS, et al. Mechanisms of cell death after pancreatic duct obstruction in the opossum and the rat. Gastroenterology. 1996;110(3):875-884.

7. van Santvoort HC, et al. Early endoscopic retrograde cholangiopancreatography in predicted severe acute biliary pancreatitis: a prospective multicenter study. Ann Surg. 2009;250(1):68-75.

8. ASGE Standards of Practice Committee, et al. Complications of ERCP. Gastrointest Endosc. 2012;75(3):467-473.

9. Malgras B, Douard R, Siauve N, Wind P. Management of left pancreatic trauma. Am Surg. 2011;77(1):1-9.
10. Coste B, et al. Piezo1 and Piezo2 are essential components of distinct mechanically activated cation channels. Science. 2010;330(6000):55-60.

11. Poole K, Herget R, Lapatsina L, Ngo HD, Lewin GR. Tuning Piezo ion channels to detect molecular-scale movements relevant for fine touch. Nat Commun. 2014;5:3520.

12. Ranade SS, et al. Piezo1, a mechanically activated ion channel, is required for vascular development in mice. Proc Natl Acad Sci U S A. 2014;111(28):10347-10352.

13. Wang S, Chennupati R, Kaur H, Iring A, Wettschureck N, Offermanns S. Endothelial cation channel PIEZO1 controls blood pressure by mediating flow-induced ATP release. J Clin Invest. 2016;126(12):4527-4536.

14. Syeda R, et al. Piezo1 channels are inherently mechanosensitive. Cell Rep. 2016;17(7):1739-1746.

15. Syeda R, et al. Chemical activation of the mechanotransduction channel Piezo1. Elife. 2015;4:e07369.

16. Li J, et al. Piezo1 integration of vascular architecture with physiological force. Nature. 2014;515(7526):279-282.

17. Miyamoto T, et al. Functional role for Piezo1 in stretch-evoked $\mathrm{Ca}^{2}(+)$ influx and ATP release in urothelial cell cultures. J Biol Chem. 2014;289(23):16565-16575.

18. Petersen OH, Ueda N. Pancreatic acinar cells: the role of calcium in stimulus-secretion coupling. J Physiol (Lond). 1976;254(3):583-606.

19. Williams JA. Regulation of pancreatic acinar cell function by intracellular calcium. Am J Physiol. 1980;238(4):G269-G279.

20. Gallacher DV, Hanley MR, Petersen OH, Roberts ML, Squire-Pollard LG, Yule DI. Substance P and bombesin elevate cytosolic $\mathrm{Ca} 2+$ by different molecular mechanisms in a rat pancreatic acinar cell line. J Physiol (Lond). 1990;426:193-207.

21. Iwatsuki N, Petersen $\mathrm{OH}$. In vitro action of bombesin on amylase secretion, membrane potential, and membrane resistance in rat and mouse pancreatic acinar cells. A comparison with other secretagogues. JClin Invest. 1978;61(1):41-46.

22. Chandra R, Liddle RA. Regulation of pancreatic secretion. Pancreapedia: Exocrine Knowledge Base. http://dx.doi.org/10.3998/panc.2015.38. Originally posted July 14, 2015. Accessed February 27, 2020.

23. Gerasimenko JV, Gerasimenko OV, Petersen OH. The role of $\mathrm{Ca} 2+$ in the pathophysiology of pancreatitis. J Physiol (Lond). 2014;592(2):269-280.

24. Gerasimenko JV, et al. Ca2+ release-activated $\mathrm{Ca} 2+$ channel blockade as a potential tool in antipancreatitis therapy. Proc Natl Acad Sci U S A. 2013;110(32):13186-13191.

25. Lerch MM, Halangk W, Mayerle J. Preventing pancreatitis by protecting the mitochondrial permeability transition pore. Gastroenterology. 2013;144(2):265-269.

26. Vonlaufen A, et al. Withdrawal of alcohol promotes regression while continued alcohol intake promotes persistence of LPS-induced pancreatic injury in alcohol-fed rats. Gut. 2011;60(2):238-246. 
27. Booth DM, et al. Reactive oxygen species induced by bile acid induce apoptosis and protect against necrosis in pancreatic acinar cells. Gastroenterology. 2011;140(7):2116-2125.

28. Huang W, et al. Fatty acid ethyl ester synthase inhibition ameliorates ethanol-induced $\mathrm{Ca} 2+-\mathrm{de}$ pendent mitochondrial dysfunction and acute pancreatitis. Gut. 2014;63(8):1313-1324.

29. Shalbueva N, et al. Effects of oxidative alcohol metabolism on the mitochondrial permeability transition pore and necrosis in a mouse model of alcoholic pancreatitis. Gastroenterology. 2013;144(2):437-446.e6.

30. Friedrich EE, et al. Endothelial cell Piezo1 mediates pressure-induced lung vascular hyperpermeability via disruption of adherens junctions. Proc Natl Acad Sci U S A . 2019;116(26):12980-12985.

31. Everaerts W, et al. Inhibition of the cation channel TRPV4 improves bladder function in mice and rats with cyclophosphamide-induced cystitis. Proc Natl Acad Sci U S A. 2010;107(44):19084-19089.

32. Sonkusare SK, et al. Elementary Ca2+ signals through endothelial TRPV4 channels regulate vascular function. Science. 2012;336(6081):597-601.

33. Thorneloe KS, et al. An orally active TRPV4 channel blocker prevents and resolves pulmonary edema induced by heart failure. Sci Transl Med. 2012;4(159):159ra148.

34. Mochizuki T, et al. The TRPV 4 cation channel mediates stretch-evoked $\mathrm{Ca} 2+$ influx and ATP release in primary urothelial cell cultures. J Biol Chem. 2009;284(32):21257-21264.

35. Pochynyuk O, Zaika O, O'Neil RG, Mamenko M. Novel insights into TRPV4 function in the kidney. Pflugers Arch. 2013;465(2):177-186

36 . Vergnolle $\mathrm{N}$, et al. A role for transient receptor potential vanilloid 4 in tonicity-induced neurogenic inflammation. Br J Pharmacol. 2010;159(5):1161-1173.

37. Andrade YN, et al. TRPV4 channel is involved in the coupling of fluid viscosity changes to epithelial ciliary activity. JCell Biol. 2005;168(6):869-874.

38. Alessandri-Haber N, Dina OA, Chen X, Levine JD. TRPC1 and TRPC6 channels cooperate with TRPV4 to mediate mechanical hyperalgesia and nociceptor sensitization. J Neurosci. 2009;29(19):6217-6228

39. Rahaman SO, et al. TRPV4 mediates myofibroblast differentiation and pulmonary fibrosis in mice. J Clin Invest. 2014;124(12):5225-5238.

40. Hof T, Chaigne S, Récalde A, Sallé L, Brette F, Guinamard R. Transient receptor potential channels in cardiac health and disease. Nat Rev Cardiol. 2019;16(6):344-360.

41. Ranade SS, Syeda R, Patapoutian A. Mechanically activated ion channels. Neuron. 2015;87(6):1162-1179.

42. Vriens J, Watanabe H, Janssens A, Droogmans G, Voets T, Nilius B. Cell swelling, heat, and chemical agonists use distinct pathways for the activation of the cation channel TRPV4. Proc Natl Acad Sci U S A. 2004;101(1):396-401.

43. Watanabe H, Vriens J, Prenen J, Droogmans G, Voets T, Nilius B. Anandamide and arachidonic acid use epoxyeicosatrienoic acids to activate TRPV4 channels. Nature. 2003;424(6947):434-438.

44. Fan HC, Zhang X, McNaughton PA. Activation of the TRPV4 ion channel is enhanced by phosphorylation. J Biol Chem. 2009;284(41):27884-27891.

45. Cao S, et al. Transient receptor potential vanilloid 4 (TRPV4) activation by arachidonic acid requires protein kinase A-mediated phosphorylation. J Biol Chem. 2018;293(14):5307-5322.

46. Bae C, Sachs F, Gottlieb PA. The mechanosensitive ion channel Piezo1 is inhibited by the peptide GsMTx4. Biochemistry. 2011;50(29):6295-6300.

47. Nathan JD, Romac J, Peng RY, Peyton M, Macdonald RJ, Liddle RA. Transgenic expression of pancreatic secretory trypsin inhibitor-I ameliorates secretagogue-induced pancreatitis in mice. Gastroenterology. 2005;128(3):717-727.

48. Gukovskaya AS, Gukovsky I, Jung Y, Mouria M, Pandol SJ. Cholecystokinin induces caspase activation and mitochondrial dysfunction in pancreatic acinar cells. Roles in cell injury processes of pancreatitis. J Biol Chem. 2002;277(25):22595-22604.

49. Lugea A, et al. Human pancreatic acinar cells: proteomic characterization, physiologic responses, and organellar disorders in ex vivo pancreatitis. Am J Pathol. 2017;187(12):2726-2743.

50. Pandol SJ, Schoeffield MS, Sachs G, Muallem $\mathrm{S}$. Role of free cytosolic calcium in secretagogue-stimulated amylase release from dispersed acini from guinea pig pancreas. J Biol Chem. 1985;260(18):10081-10086.

51. Pandol SJ, Schoeffield MS, Fimmel CJ, Muallem $\mathrm{S}$. The agonist-sensitive calcium pool in the pancreatic acinar cell. Activation of plasma membrane Ca2+ influx mechanism. J Biol Chem. 1987;262(35):16963-16968.

52. Raraty M, et al. Calcium-dependent enzyme activation and vacuole formation in the apical granular region of pancreatic acinar cells. Proc Natl Acad Sci U S A . 2000;97(24):13126-13131.

53. Walsh DWM, et al. Live cell imaging of mitochondria following targeted irradiation in situ reveals rapid and highly localized loss of membrane potential. Sci Rep. 2017;7:46684.

54. Lerch MM, Gorelick FS. Early trypsinogen activation in acute pancreatitis. Med Clin North Am. 2000;84(3):549-563, viii.

55. Gerasimenko JV, et al. Calmodulin protects against alcohol-induced pancreatic trypsinogen activation elicited via $\mathrm{Ca} 2+$ release through IP3 receptors. Proc Natl Acad Sci U S A. 2011;108(14):5873-5878.

56. Halestrap AP, Richardson AP. The mitochondrial permeability transition: a current perspective on its identity and role in ischaemia/reperfusion injury. J Mol Cell Cardiol. 2015;78:129-141.

57. Bagher P, Beleznai T, Kansui Y, Mitchell R, Garland CJ, Dora KA. Low intravascular pressure activates endothelial cell TRPV4 channels, local $\mathrm{Ca} 2+$ events, and IKCa channels, reducing arteriolar tone. Proc Natl Acad Sci U S A 2012;109(44):18174-18179.

58. Hamaguchi $\mathrm{K}$, et al. Induction of distinct sets of secretory phospholipase $\mathrm{A}(2)$ in rodents during inflammation. Biochim Biophys Acta. 2003;1635(1):37-47.

59. Riendeau D, et al. Arachidonyl trifluoromethyl ketone, a potent inhibitor of $85-\mathrm{kDa}$ phospholipase A2, blocks production of arachidonate and 12-hydroxyeicosatetraenoic acid by calcium ionophore-challenged platelets. J Biol Chem.
1994;269(22):15619-15624.

60. Criddle DN, et al. Calcium signalling and pancreatic cell death: apoptosis or necrosis? Cell Death Differ. 2007;14(7):1285-1294.

61. Acosta JM, Ledesma CL. Gallstone migration as a cause of acute pancreatitis. $N$ Engl J Med. 1974;290(9):484-487.

62. Neoptolemos JP, Carr-Locke DL, London N, Bailey I, Fossard DP. ERCP findings and the role of endoscopic sphincterotomy in acute gallstone pancreatitis. Br J Surg. 1988;75(10):954-960.

63. Bradley EL. Pancreatic duct pressure in chronic pancreatitis. Am J Surg. 1982;144(3):313-316

64. Alper SL. Genetic Diseases of PIEZO1 and PIEZO2 Dysfunction. Curr Top Membr. 2017;79:97-134.

65. Lewis AH, Cui AF, McDonald MF, Grandl J. Transduction of repetitive mechanical stimuli by Piezo1 and Piezo2 ion channels. Cell Rep. 2017;19(12):2572-2585

66. Petersen $\mathrm{OH}$. Ca2+-induced pancreatic cell death: roles of the endoplasmic reticulum, zymogen granules, lysosomes and endosomes. J Gastroenterol Hepatol. 2008;23 Suppl 1:S31-S36.

67. Li J, Zhou R, Zhang J, Li ZF. Calcium signaling of pancreatic acinar cells in the pathogenesis of pancreatitis. World J Gastroenterol. 2014;20(43):16146-16152.

68. Petersen OH, Tepikin AV. Polarized calcium signaling in exocrine gland cells. Annu Rev Physiol. 2008;70:273-299.

69. Voronina SG, Gryshchenko OV, Gerasimenko OV, Green AK, Petersen OH, Tepikin AV. Bile acids induce a cationic current, depolarizing pancreatic acinar cells and increasing the intracellular $\mathrm{Na}+$ concentration. J Biol Chem. 2005;280(3):1764-1770.

70. Pandol SJ, Saluja AK, Imrie CW, Banks PA. Acute pancreatitis: bench to the bedside. Gastroenterol ogy. 2007;132(3):1127-1151.

71. Criddle DN, et al. Fatty acid ethyl esters cause pancreatic calcium toxicity via inositol trisphosphate receptors and loss of ATP synthesis. Gastroenterology. 2006;130(3):781-793.

72. Leach SD, Modlin IM, Scheele GA, Gorelick FS. Intracellular activation of digestive zymogens in rat pancreatic acini. Stimulation by high doses of cholecystokinin. J Clin Invest. 1991;87(1):362-366.

73. Criddle DN, McLaughlin E, Murphy JA, Petersen $\mathrm{OH}$, Sutton R. The pancreas misled: signals to pancreatitis. Pancreatology. 2007;7(5-6):436-446.

74. Mukherjee R, et al. Mechanism of mitochondrial permeability transition pore induction and damage in the pancreas: inhibition prevents acute pancreatitis by protecting production of ATP. Gut. 2016;65(8):1333-1346.

75. Alavian KN, et al. An uncoupling channel within the c-subunit ring of the F1FO ATP synthase is the mitochondrial permeability transition pore. Proc Natl Acad Sci U S A. 2014;111(29):10580-10585.

76. Giorgio V, et al. Dimers of mitochondrial ATP synthase form the permeability transition pore. Proc Natl Acad Sci U S A. 2013;110(15):5887-5892.

77. Elrod JW, et al. Cyclophilin D controls mitochondrial pore-dependent $\mathrm{Ca}(2+)$ exchange, metabolic flexibility, and propensity for heart failure in mice. J Clin Invest. 2010;120(10):3680-3687.

78. Saluja AK, Bhagat L, Lee HS, Bhatia M, Frossard 
JL, Steer ML. Secretagogue-induced digestive enzyme activation and cell injury in rat pancreatic acini. Am J Physiol. 1999;276(4):G835-G842.

79. Mooren FCh, et al. Early changes in pancreatic acinar cell calcium signaling after pancreatic duct obstruction. J Biol Chem. 2003;278(11):9361-9369.

80. Watanabe H, Vriens J, Suh SH, Benham CD, Droogmans G, Nilius B. Heat-evoked activation of TRPV4 channels in a HEK293 cell expression system and in native mouse aorta endothelial cells. J Biol Chem. 2002;277(49):47044-47051.

81. Yule DI. Ca2+ Signaling in Pancreatic Acinar Cells. Pancreapedia: Exocrine Pancreas Knowledge Base. http://dx.doi.org/10.3998/panc.Originally posted July 14, 2015. Accessed February 27, 2020.

82. Cosen-Binker LI, Gaisano HY. Recent insights into the cellular mechanisms of acute pancreati- tis. Can J Gastroenterol. 2007;21(1):19-24.

83. Sendler M, et al. Cathepsin B-mediated activation of trypsinogen in endocytosing macrophages increases severity of pancreatitis in mice. Gastroenterology. 2018;154(3):704-718.e10.

84. Cahalan SM, Lukacs V, Ranade SS, Chien S, Bandell M, Patapoutian A. Piezo1 links mechanical forces to red blood cell volume. Elife. 2015;4:e07370.

85. Kanju P, et al. Small molecule dual-inhibitors of TRPV4 and TRPA1 for attenuation of inflammation and pain. Sci Rep. 2016;6:26894.

86. Liddle RA, Goldfine ID, Williams JA. Bioassay of plasma cholecystokinin in rats: effects of food, trypsin inhibitor, and alcohol. Gastroenterology. 1984;87(3):542-549.

87. Williams JA. Isolation of rodent pancreatic acinar cells and acini by collagenase digestion. Pancreapedia: Exocrine Pancreas Knowledge Base. http://dx. doi.org/10.3998/panc.2010.18. Originally posted November 13, 2010. Accessed February 27, 2020.

88. Li J, et al. Circulating fibrocytes stabilize blood vessels during angiogenesis in a paracrine manner. Am J Pathol. 2014;184(2):556-571.

89. De Groef S, et al. Surgical injury to the mouse pancreas through ligation of the pancreatic duct as a model for endocrine and exocrine reprogramming and proliferation. JVis Exp. 2015;(102):e52765.

90. Sendler M, et al. Complement component 5 mediates development of fibrosis, via activation of stellate cells, in 2 mouse models of chronic pancreatitis. Gastroenterology. 2015;149(3):765-76.e10.

91. Spormann H, Sokolowski A, Letko G. Effect of temporary ischemia upon development and histological patterns of acute pancreatitis in the rat. Pathol Res Pract.1989;184(5):507-513. 